\title{
The homotopy dimension of codiscrete subsets of the 2-sphere $\mathbb{S}^{2}$
}

\author{
by \\ J. W. Cannon and G. R. Conner (Provo, UT) \\ Dedicated to the memory of Karol Borsuk \\ on the occasion of the centennial of his birth
}

\begin{abstract}
Andreas Zastrow conjectured, and Cannon-Conner-Zastrow proved, that filling one hole in the Sierpiński curve with a disk results in a planar Peano continuum that is not homotopy equivalent to a 1-dimensional set. Zastrow's example is the motivation for this paper, where we characterize those planar Peano continua that are homotopy equivalent to 1-dimensional sets.

While many planar Peano continua are not homotopy equivalent to 1-dimensional compacta, we prove that each has fundamental group that embeds in the fundamental group of a 1-dimensional planar Peano continuum.

We leave open the following question: Is a planar Peano continuum homotopically 1-dimensional if its fundamental group is isomorphic with the fundamental group of a 1-dimensional planar Peano continuum?
\end{abstract}

1. Introduction. We say that a subset $X$ of the 2 -sphere $\mathbb{S}^{2}$ is codiscrete if its complement $D(X)$, as subspace of $\mathbb{S}^{2}$, is discrete. For a codiscrete set $X$, the set $B(X)$ of limit points of $D(X)$ in $\mathbb{S}^{2}$, which is necessarily a closed subset of $X$ having dimension $\leq 1$, is called the bad set of $X$. The homotopy dimension of a space $X$ is the smallest dimension of a space homotopy equivalent to $X$. We say that $X$ is homotopically $\leq k$-dimensional if its homotopy dimension is $\leq k$. Our main theorem characterizes the homotopy dimension of $X$ in terms of the interplay between $D(X)$ and $B(X)$ :

TheOREM 1.1 (Characterization Theorem). Suppose that $X$ is a codiscrete subset of the 2 -sphere $\mathbb{S}^{2}$. Then $X$ has homotopy dimension $\leq 1$ if and only if the following two conditions are satisfied:

2000 Mathematics Subject Classification: Primary 57N05; Secondary 54F45, 54F50, $55 \mathrm{M} 10,55 \mathrm{P} 10$.

Key words and phrases: Peano continuum, codiscrete subset, homotopy dimension.

The research of the first author was supported NSF research grant DMS-10104030. 
(1) Every component of $\mathbb{S}^{2} \backslash B(X)$ contains a point of $D(X)$.

(2) If $D$ is any closed disk in the 2-sphere $\mathbb{S}^{2}$, then the components of $D \backslash B(X)$ that do not contain any point of $D(X)$ form a null sequence.

[Recall that a sequence $C_{1}, C_{2}, \ldots$ is a null sequence if the diameters of the sets $C_{n}$ approach 0 as $n$ approaches $\infty$.] Examples appear in the figure. The first two examples of possible bad sets are locally connected. The one is a circle with a null sequence of Hawaiian earrings attached. The other is a Sierpiński curve. The associated codiscrete set will be homotopically $\leq 1$ dimensional if and only if condition (1) is satisfied. The third example gives an example of a possible bad set that is not locally connected. In order that the associated codiscrete set be homotopically 1-dimensional, both conditions (1) and (2) must be satisfied. Thus, near each point of the limiting arc, where any small disk is separated into infinitely many components by the oscillating curve, almost all of these components must contain a point of $D(X)$.

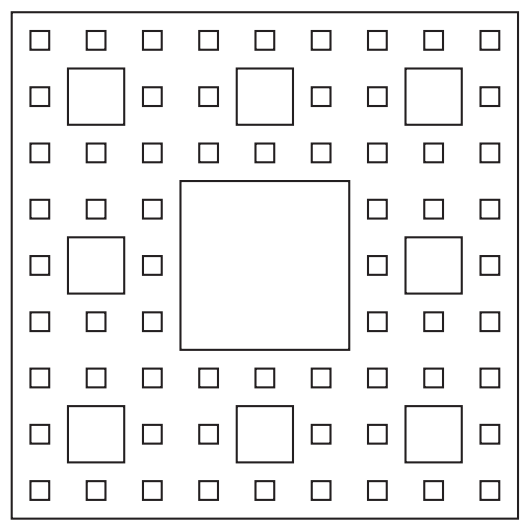

Sierpiński curve

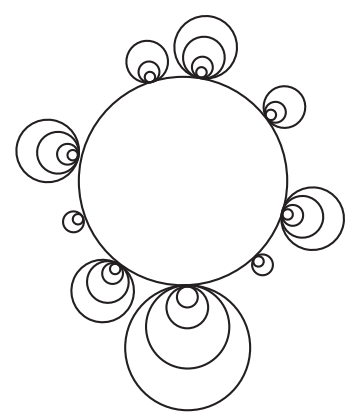

Circle wedged with a countable null sequence of Hawaiian earrings

Locally connected bad sets

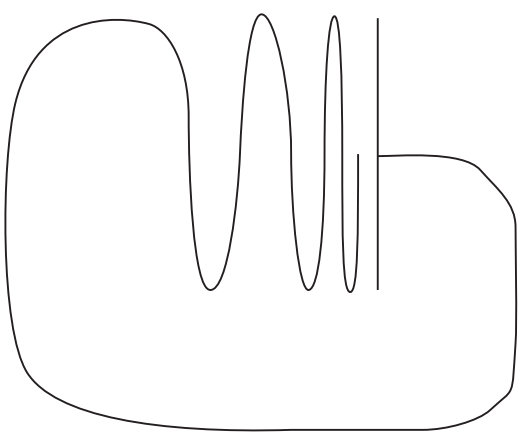

A non-locally-connected bad set: the Warsaw circle 
A compactum is a compact metric space. A continuum is a connected compactum. A Peano continuum is a locally connected continuum; equivalently, a Peano continuum is the metric continuous image of the interval $[0,1]$. 1.1 applies to all Peano continua in the 2-sphere $\mathbb{S}^{2}$ because of the following well-known theorem:

TheOREM 1.2. Every Peano continuum $M$ in the 2 -sphere $\mathbb{S}^{2}$ is homotopy equivalent to a codiscrete subset $X$ of $\mathbb{S}^{2}$. Conversely, every codiscrete subset $X$ of $\mathbb{S}^{2}$ is homotopy equivalent to a Peano continuum $M$ in $\mathbb{S}^{2}$.

We shall indicate later (after Theorem 2.9) how this well-known theorem is proved. For the moment, we simply mention that, given $M$, one can obtain an appropriate codiscrete subset $X$ by choosing for $D(X)$ exactly one point from each component of $\mathbb{S}^{2} \backslash M$. One can define the bad set $B(M)$ of $M$ as the bad set $B(X)$ of $X$. It is natural to ask how restricted bad sets are. The following theorem, which characterizes the possible bad sets of codiscrete sets $X$, is actually an easy exercise which we leave to the reader:

TheOREM 1.3. A subset $B$ of the 2-sphere $\mathbb{S}^{2}$ is the bad set $B(X)$ of some codiscrete subset $X \subset \mathbb{S}^{2}$ if and only if $B$ is closed and has dimension less than 2.

It is an easy matter to use Characterization Theorem 1.1 and the construction inherent in Theorem 1.3 to construct all manner of interesting planar Peano continua that are, or are not, homotopy equivalent to a 1dimensional set. All the examples that have appeared in the literature (see [4] and [12]) are likewise easily checked by means of Characterization Theorem 1.1 .

In light of the fact that so many planar Peano continua are not homotopically 1-dimensional, it is a little surprising to find that their fundamental groups are essentially 1-dimensional in the following sense:

THEOREM 1.4. If $M$ is a planar Peano continuum, then the fundamental group of $M$ embeds in the fundamental group of a 1-dimensional planar Peano continuum.

Corollary 1.5. If $M$ is a planar Peano continuum, then the fundamental group of $M$ embeds in an inverse limit of finitely generated free groups.

Question 1.6. If $M$ is a planar Peano continuum whose fundamental group is isomorphic with the fundamental group of some 1-dimensional planar Peano continuum, is it true that $M$ is homotopically 1-dimensional?

The remaining sections of this paper will be devoted to proofs of these theorems. 
2. Fundamental ideas and tools. Our proofs make substantial use of classical topology of the plane. As good basic references we suggest [21], [19], [20], and [10], all deriving their initial impetus from the school and work of R. L. Moore, who summarized much of his work in [15].

To minimize the necessity of turning to these references, we collect here a number of the basic ideas and tools that will be used often in the proofs. Many of these will be familiar to some of our readers. The topics will be outlined in bold type so that the reader can quickly find those topics with which they are not familiar. For many, the best way to read the paper will be to turn immediately to the later sections and return to this section only when they encounter a tool or idea with which they are not familiar. Where it is possible, we outline the proofs so that the reader will not have to hunt for obscure references.

Our first fundamental idea is that the Tietze Extension Theorem can be used to cut off a map $f$ on an absolute retract $R$. Let $f: X \rightarrow Y$ be a continuous function from a compactum $X$ to a space $Y$. Assume that $R$ is a closed subset of $Y$, that $R$ is an absolute retract, and that $f^{-1}(R)$ separates $X$ into disjoint open subsets $A$ and $B$. Then we obtain a new function $f^{\prime}: X \rightarrow Y$ as follows: We define $f^{\prime}\left|\left(A \cup f^{-1}(R)\right)=f\right|\left(A \cup f^{-1}(R)\right)$. Since $R$ is an absolute retract and $f^{-1}(R)$ is a closed subspace of the normal space $B \cup f^{-1}(R)$, there is a continuous function $\left.f^{\prime} \mid B \cup f^{-1}(R)\right)$ that extends $f \mid f^{-1}(R)$ and takes $B \cup f^{-1}(R)$ into $R$. We say that the map $f^{\prime}$ cuts $f$ off on $R$, fixing $A$.

We next consider the Phragmén-Brouwer properties, all satisfied by the $n$-sphere $\mathbb{S}^{n}$ for $n \geq 2$. R. L. Wilder, in [21, Chapter 2, Theorem 4.12], proves the equivalence of seven of them ([21, pp. 47-50]) in metric spaces $S$ that are connected and locally connected. We mention only two:

Property I. If $A$ and $B$ are disjoint closed subsets of $S$, and $x, y \in S$ are such that neither $A$ nor $B$ separates $x$ and $y$ in $S$, then $A \cup B$ does not separate $x$ and $y$ in $S$.

Property II (Brouwer Property). If $M$ is a closed, connected subset of $S$ and $C$ is a component of $S \backslash M$, then the boundary of $C$ is a closed and connected set.

Here are two corollaries of Property I:

Corollary 2.1. If $A$ is an annulus and $C$ is a closed subset of $\operatorname{int}(A)$ that separates the boundary components $J_{1}$ and $J_{2}$ of $A$, then some component of $C$ separates $J_{1}$ from $J_{2}$ in $A$.

Corollary 2.2. If $D$ is the square disk and $C$ is a closed subset of $D$ that separates the top of $D$ from the bottom of $D$, then some component of $C$ separates them. 
The first of these corollaries is an immediate application of Property I, when one passes to a minimal separator (which exists by the Brouwer Reduction Theorem, [20, Chapter I, 11.1]). The second follows from the first when one fixes the right and left sides of $D$ and doubles the remainder of $D$ to form an annulus.

We apply these ideas to show that homotopies of $X$ within itself must fix the bad set $B(X)$ pointwise. This general principle can be applied to all connected planar sets $X$ and not just to codiscrete sets. If $X$ is any connected planar set, then we may define the bad set $B(X)$ of $X$ to be the set of points $x \in X$ having the property that, in each neighborhood of $x$, there is a simple closed curve $J$ in $X$ such that the interior of $J$ in the plane $\mathbb{R}^{2}$ is not entirely contained in the set $X$. This modified definition coincides with the previous definition when the set $X$ is codiscrete.

Theorem 2.3 ([4, Theorem 5.2]). Suppose that $X$ is a connected planar set and that $x \in B(X)$. Then every homotopy of $X$ within $X$ fixes the point $x$.

Proof. Suppose that there is a homotopy $H: X \times[0,1] \rightarrow X$ such that $H(y, 0)=y$ for all $y \in X$ and $H(x, 1) \neq x$. Let $N_{0}$ and $N_{1}$ be disjoint neighborhoods of $x$ and $H(x, 1)$, respectively. By continuity, there is a neighborhood $M$ of $x$ in $N_{0}$ such that $H(M, 1) \subset N_{1}$. There is a round circle $J$ around $x$ that is not contained in $X$ but intersects $X$ only in $N_{0}$. There is a simple closed curve $K$ in $\operatorname{int}(J) \cap M \subset X$ whose interior is not contained entirely in $X$. By 2.1, the annulus $K \times[0,1]$ has its boundary components separated by the $H$-preimage of some component of $J \cap X$. This component maps into a single component $L$ of $J \cap X$. Since $L$ is an absolute retract that is closed in $X$, the homotopy $H \mid K \times[0,1]$ can be cut off at this component via the Tietze Extension Theorem, fixing $H \mid K \times\{0\}$. The image of of $K \times\{1\}$ in $L$ is nullhomotopic in $L$. This allows one to shrink $K$ in $X$, an impossibility.

Our next fundamental idea is that of the convergence of a sequence of sets. The two books by Whyburn, [19] and [20], and the text by Hocking and Young, [10], give a good treatment of this topic. However, it does not take much space to review the basics here. Suppose that $A_{1}, A_{2}, \ldots$ is a sequence of subsets of a space $S$. We say that a point $x \in S$ is an element of $\liminf _{i} A_{i}$ if every neighborhood of $x$ intersects all but finitely many of the sets $A_{i}$. We say that $x$ is an element of $\lim _{\sup _{i}} A_{i}$ if every neighborhood of $x$ intersects infinitely many of the sets $A_{i}$. We say that the sequence $A_{i}$ converges if the lim inf and limsup coincide. The limit is defined to be this common liminf and limsup.

Theorem 2.4 ([19, Chapter I, Theorem 7.1]; [20, Chapter I, Theorem 7.1]; [10, pp. 102-103]). If $A_{1}, A_{2}, \ldots$ is any sequence of sets in a separable metric space $S$, then there is a convergent subsequence. 
Proof. Let $U_{1}, U_{2}, \ldots$ be a countable basis for the topology of $S$. Let $S_{0}$ be the given sequence $A_{1}, A_{2}, \ldots$ of subsets of the space $S$. Assume inductively that a subsequence $S_{i}$ of $S$ has been chosen. If there is a subsequence of $S_{i}$ no element of which intersects $U_{i+1}$, let $S_{i+1}$ be such a subsequence. Otherwise, let $S_{i+1}=S_{i}$. Let $S_{\infty}$ be the diagonal sequence, which takes as first element the first element of $S_{1}$, as second element the second element of $S_{2}$, etc. We claim that the subsequence $S_{\infty}$ of $S_{0}$ converges. Indeed, suppose that $x \in \lim \sup S_{\infty}$, that is, every neighborhood of $x$ intersects infinitely many elements of $S_{\infty}$. Suppose that there is a neighborhood $U_{j}$ of $x$ that misses infinitely many elements of $S_{\infty}$. Then $S_{j}$, by definition, must miss $U_{j}$. But this implies that all elements of $S_{\infty}$ with index as high as $j$ miss $U_{j}$, a contradiction. Thus, every element of the limsup lies in the liminf. Since the opposite inclusion is obvious, these two limits are equal, and the sequence $S_{\infty}$ converges.

Theorem 2.5 (Properties of the limit of a convergent sequence). Suppose that the sequence $A_{1}, A_{2}, \ldots$ of nonempty subsets of a separable metric space $S$ converges to a set $A$. Then

(1) the set $A$ is closed in $S$;

(2) if $S$ is compact, then $A$ is nonempty and compact;

(3) if $S$ is compact and if each $A_{i}$ is connected, then the limit $A$ is nonempty, compact, and connected;

(4) if $S$ is compact and if each $A_{i}$ has diameter $\geq \varepsilon$, then $A$ has diameter $\geq \varepsilon$.

Proof. Easy exercise.

We shall in more than one place make use of R. L. Moore's Decomposition Theorem. In 1919 [13], R. L. Moore characterized the Euclidean plane topologically. In 1925 [14], he noted that his axioms were also satisfied by a large class of quotient spaces of the plane, so that those identification spaces were also planes.

Since Moore's theorem is somewhat inaccessible to today's readers because of evolving terminology and background, we will give a fairly straightforward statement and we will outline the proof of this theorem. In the remarks following the statement of the theorem, we point out some equivalent statements of the fundamental hypotheses of the theorem that we use in our applications. If any of the equivalent hypotheses is satisfied, we shall simply refer to the Moore Decomposition Theorem.

Theorem 2.6 (Moore Decomposition Theorem). Suppose that $f: \mathbb{S}^{2} \rightarrow X$ is a continuous map from the 2 -sphere $\mathbb{S}^{2}$ onto a Hausdorff space $X$ such that, for each $x \in X$, the set $\mathbb{S}^{2} \backslash f^{-1}(x)$ is homeomorphic with the plane $\mathbb{R}^{2}$. Then $X$ is a 2-sphere. 
REMARKS. (1) The requirement that $\mathbb{S}^{2} \backslash f^{-1}(x)$ be homeomorphic with $\mathbb{R}^{2}$ is equivalent to the requirement that both $f^{-1}(x)$ and $\mathbb{S}^{2} \backslash f^{-1}(x)$ be nonempty and connected.

(2) The Hausdorff condition is equivalent in this setting to the more central requirement that $f$ be a closed map. The map $f$ is closed if, whenever $C \subset \mathbb{S}^{2}$ is closed in $\mathbb{S}^{2}$, the set $f(C)$ is closed in $X$. This, of course, in light of the surjectivity of $f$, makes $f$ an identification map.

An identification map $f: A \rightarrow B$ (surjective by hypothesis) is closed if and only if the collection $G=\left\{f^{-1}(b) \mid b \in B\right\}$ satisfies the upper semicontinuity condition, which states that, if $U$ is an open subset of $A$, then the union of the elements of $G$ that lie in $U$ is also an open subset of $A$.

Moore originally stated his theorem in terms of upper semicontinuous collections $G$ filling $\mathbb{S}^{2}$ or $\mathbb{R}^{2}$ and described the topology of the resulting quotient spaces $\mathbb{S}^{2} / G$ and $\mathbb{R}^{2} / G$. The elements of $G$ are simply the point preimages of the surjection $f$.

The Hausdorff condition of the theorem can often be checked by considering sequences of elements of $G$ and their limit points. A sequence $\left\{g_{i}\right\}$ of elements of $G$ is not allowed to contain convergent point sequences $\left\{x_{i}\right\}$ and $\left\{y_{i}\right\}$ with limit points $x$ and $y$ in two different elements $h$ and $k$ of $G$, for then one would not be able to separate in $X$ the points defined by $h$ and $k$. The upper semicontinuity requirement is always satisfied when the nondegenerate elements of $G$ form a null sequence.

(3) The R. L. Moore Theorem has local versions that allow generalizations to other 2-manifolds.

(4) There is an easy analogous version for quotients of the circle, where point preimages are points or (closed) arcs.

(5) The theorem has generalizations to higher dimensions that require stronger hypotheses: Suppose that $f: \mathbb{S}^{n} \rightarrow X$ is a continuous map from the $n$-sphere $\mathbb{S}^{n}$ onto a finite-dimensional Hausdorff space $X$ such that, for each $x \in X$, the set $\mathbb{S}^{n} \backslash f^{-1}(x)$ is homeomorphic with the Euclidean space $\mathbb{R}^{n}$. Then $X$ is an $n$-sphere provided that, in addition, $n \geq 5$, and $X$ satisfies the condition that maps $g: \mathbb{B}^{2} \rightarrow X$ from the 2 -dimensional disk $\mathbb{B}^{2}$ into $X$ can be approximated by embeddings. This generalization was conjectured and proved in many special cases by Cannon (see [2] for a substantial discussion of these matters) and proved in general by R. D. Edwards (see Daverman's book [7]). The situation in dimensions 3 and 4 has not been completely resolved.

The proof of Moore's Theorem 2.6, which we shall outline, relies on a more intuitive theorem, called the Zippin Characterization Theorem. (See, for example, [21, Chapter III, Theorem 4.2].)

TheOREM 2.7 (Zippin Characterization Theorem). The space $X$ is a 2-sphere if the following four conditions are satisfied: 
(i) $X$ is a nondegenerate Peano continuum.

(ii) No point $x \in X$ separates $X$ (so that, in particular, $X$ contains at least one simple closed curve).

(iii) Each simple closed curve $J \subset X$ separates $X$.

(iv) No arc $A \subset X$ separates $X$.

Proof of Theorem 2.6. We prove the Moore Decomposition Theorem on the basis of the Zippin Characterization Theorem. We verify the four conditions of the Zippin Theorem in turn. (Note that conditions (iii) and (iv) are true in the 2 -sphere by standard homological arguments. We shall use those same arguments here.)

(i) Since $X$ is Hausdorff, the map $f$ is a closed surjection; hence it is easy to verify the conditions of the Urysohn metrization theorem so that $X$ is metric. (See [16, Theorem 34.1].) Since $\mathbb{S}^{2}$ is a Peano continuum, that is, a metric continuous image of $[0,1]$, so also is $X$. Since, for all $x \in X$, both $f^{-1}(x)$ and $\mathbb{S}^{2} \backslash f^{-1}(x)$ are nonempty, $X$ has more than one point; that is, $X$ is nondegenerate.

(ii) By hypothesis, $\mathbb{S}^{2} \backslash f^{-1}(x)$ is connected. Hence so is $X \backslash\{x\}=$ $f\left(\mathbb{S}^{2} \backslash f^{-1}(x)\right)$.

(iii) Let $p_{1}, p_{2} \in J$ cut $J$ into two arcs $A_{1}$ and $A_{2}$. Then $f^{-1}\left(A_{1}\right)$ and $f^{-1}\left(A_{2}\right)$ are compact, connected, and have nonconnected intersection $f^{-1}\left(p_{1}\right) \cup f^{-1}\left(p_{2}\right)$. The reduced Mayer-Vietoris homology sequence for the pair $U=\mathbb{S}^{2} \backslash f^{-1}\left(A_{1}\right)$ and $V=\mathbb{S}^{2} \backslash f^{-1}\left(A_{2}\right)$ contains the segment

$$
\begin{aligned}
H_{1}\left(\mathbb{S}^{2} \backslash f^{-1}\left(A_{1}\right)\right) & \oplus H_{1}\left(\mathbb{S}^{2} \backslash f^{-1}\left(A_{2}\right)\right) \\
& \rightarrow H_{1}\left(\mathbb{S}^{2} \backslash\left(f^{-1}\left(p_{1}\right) \cup f^{-1}\left(p_{2}\right)\right) \rightarrow \widetilde{H}_{0}\left(\mathbb{S}^{2} \backslash\left(f^{-1}(J)\right)\right),\right.
\end{aligned}
$$

where $H_{1}(U)=H_{1}(V)=0$ since $f^{-1}\left(A_{1}\right)$ and $f^{-1}\left(A_{2}\right)$ are connected and $H_{1}(U \cup V) \neq 0$ since $f^{-1}\left(A_{1}\right) \cap f^{-1}\left(A_{2}\right)$ is not connected. Thus we have $\widetilde{H}_{0}\left(\mathbb{S}^{2} \backslash f^{-1}(J)\right)=\widetilde{H}_{0}(U \cap V) \neq 0$, so that $f^{-1}(J)$ separates $\mathbb{S}^{2}$. Consequently, $J$ separates $X$.

(iv) If $p \in A$ separates $A$ into $\operatorname{arcs} A_{1}$ and $A_{2}$, and if $A$ separates $x$ and $y$ in $X$, then we claim that one of $A_{1}$ and $A_{2}$ also separates $x$ and $y$ in $X$; indeed, we see this by considering $f^{-1}(A)=f^{-1}\left(A_{1}\right) \cup f^{-1}\left(A_{2}\right)$, which must separate $f^{-1}(x)$ from $f^{-1}(y)$ in $\mathbb{S}^{2}$. The reduced Mayer-Vietoris homology sequence for the pair $U=\mathbb{S}^{2} \backslash f^{-1}\left(A_{1}\right)$ and $V=\mathbb{S}^{2} \backslash f^{-1}\left(A_{2}\right)$ contains the segment

$$
0 \rightarrow \widetilde{H}_{0}\left(\mathbb{S}^{2} \backslash f^{-1}(A)\right) \rightarrow \widetilde{H}_{0}\left(\mathbb{S}^{2} \backslash f^{-1}\left(A_{1}\right)\right) \oplus \widetilde{H}_{0}\left(\mathbb{S}^{2} \backslash f^{-1}\left(A_{2}\right)\right) .
$$

Given any $u \in f^{-1}(x)$ and $v \in f^{-1}(y)$, the element $u-v$ represents a nonzero element of the center group, hence maps to a nonzero element of $\widetilde{H}_{0}\left(\mathbb{S}^{2} \backslash f^{-1}\left(A_{1}\right) \oplus \widetilde{H}_{0}\left(\mathbb{S}^{2} \backslash f^{-1}\left(A_{2}\right)\right)\right.$. Therefore, either $f^{-1}\left(A_{1}\right)$ or $f^{-1}\left(A_{2}\right)$ separates $u$ from $v$ in $\mathbb{S}^{2}$, implying the claim. 
By induction, one obtains intervals $I_{0} \supset I_{1} \supset \cdots$ that separate $x$ and $y$ in $X$ such that $\bigcap_{n=1}^{\infty} I_{n}$ is a single point $q$ that does not separate $x$ from $y$. But an arc $\alpha$ from $x$ to $y$ in the path connected open set $X \backslash\{q\}$ misses some $I_{n}$, a contradiction. We conclude that $A$ cannot separate $X$.

The proof of the Moore Decomposition Theorem 2.6 is complete.

Our sixth topic is that of locally connected continua in the plane. The following theorem characterizes planar Peano continua in several ways, all well-known. A version of this theorem appears in [9].

THEOREM 2.8. Suppose that $M$ is a continuum (= compact, connected subset) in the 2 -sphere $\mathbb{S}^{2}$. Then $M$ is a Peano continuum (= locally connected continuum) if and only if the following four equivalent conditions are satisfied:

(1) For each disk $D$ in $\mathbb{S}^{2}$, the components of $D \backslash M$ form a null sequence.

$\left(1^{\prime}\right)$ For each disk $D$ in $\mathbb{S}^{2}$, the components of $D \cap M$ form a null sequence.

(2) For each annulus $A$ in $\mathbb{S}^{2}$, the components of $A \backslash M$ that intersect both boundary components of $A$ are finite in number.

$\left(2^{\prime}\right)$ For each annulus $A$ in $\mathbb{S}^{2}$, the components of $A \cap M$ that intersect both boundary components of $A$ are finite in number.

REMARK. All proofs involving continua $M \subset \mathbb{R}^{2}$ that are not locally connected involve the construction of limit continua, that is, nondegenerate continua $L \subset M$ that are limits of a sequence of disjoint nondegenerate continua $L_{i}$ in the complement of $M$ that are separated from one another by $M$ in an open subset of $M$.

Proof. Assume that $M$ is locally connected but (1) is not satisfied, so that, for some disk $D$ in $\mathbb{S}^{2}$, the components of $D \backslash M$ do not form a null sequence. Then some sequence $U_{i}$ of such components converges to a nondegenerate continuum $U$ in $\mathbb{S}^{2}$ by Theorems 2.4 and 2.5. Let $A$ be an annulus in $\mathbb{S}^{2}$ that separates two points of $U$. Then each $U_{i}$ contains an arc $A_{i}$ irreducibly joining the two ends of $A$. We may assume that they converge to a continuum $A^{\prime}$ joining the two ends of $A$. The continuum $A^{\prime}$ must be a subset of $M$, for otherwise it could not have points of infinitely many of the components $U_{i}$ close to it. We may pass to a subsequence of the $A_{i}$ 's so chosen that their endpoints on one boundary component $J$ of the annulus $A$ converge monotonically on $J$. It then follows that each $A_{i}$ is adjacent to $A_{i+1}$, with neither $A^{\prime}$ nor any other $A_{j}$ between them. They must therefore be separated by a component $M_{i}$ of $A \cap M$ that intersects both ends of $A$. (See Corollary 2.2.) The components $M_{i}$ converge to a subcontinuum of $A^{\prime}$ that joins the ends of $A$. This shows that $M$ is not locally connected at these points of $A^{\prime}$, a contradiction. 
Suppose (1) is satisfied but $\left(1^{\prime}\right)$ is not. That is, there is a disk $D$ in $\mathbb{S}^{2}$ and infinitely many large components of $D \cap M$. We may take a sequence of such components that converge to a nondegenerate subcontinuum of $M$. We take an annulus $A$ that separates two points of the limit continuum. Infinitely many of the large components cross this annulus. They are separated by large components of $A \backslash M$ that cross the annulus. Arcs in these components that cross the annulus allow one to form a disk $D$ that is crossed by infinitely many large components of $D \backslash M$, a contradiction to (1). We conclude that $\left(1^{\prime}\right)$ is satisfied.

Similar arguments show that $\left(1^{\prime}\right)$ implies (1) and that these are equivalent to $(2)$ and $\left(2^{\prime}\right)$.

Finally, if $M$ is not locally connected, then there is a component of an open subset of $M$ that is not open. That is, there is an open set $N$, a component $C$ of $N$, and a point $p \in C$ such that $p$ is a limit point of $N \backslash C$. If $p_{i}$ is a sequence from $N \backslash C$ converging to $p$, then no component of $N$ can contain more than finitely many of the points $p_{i}$, for, otherwise, $p$ would be a point of that component. But each component of $N$ has a limit point in $M \backslash N$ (prove as an exercise or refer to [20, Chapter I, 10.1]). Thus the closure of each of these components intersects the boundary of $N$ in $M$. These large components contradict $\left(1^{\prime}\right)$.

Theorem 2.9 (Filling Theorem). Suppose that $M$ is a Peano continuum in the 2-sphere $\mathbb{S}^{2}$, and suppose that $U$ is a component of the complement of $M$ in $\mathbb{S}^{2}$. Then there is a map $f: \mathbb{B}^{2} \rightarrow \operatorname{cl}(U)$ from the 2-disk $\mathbb{B}^{2}$ onto the closure of the domain $U$ that takes $\operatorname{int}\left(B^{2}\right)$ homeomorphically onto $U$ and takes $\mathbb{S}^{1}=\partial\left(\mathbb{B}^{2}\right)$ continuously onto $\partial(U)$. In addition, if $A$ is a free boundary arc of $\operatorname{cl}(U)$, then we may assume that the map $f$ is one-to-one over the arc $A$. Furthermore, we may assume that, for each $p \in \mathbb{S}^{1}$, the set $f^{-1} f(p)$ is totally disconnected.

Remark. That the arc $A$ is free means that $A$ is accessible from precisely one of its sides from the domain $U$ and that $\operatorname{int}(A)$ is an open subset of $\partial(U)$.

Proof. We give only an indication of the proof. There are well-known, completely topological proofs of this theorem. However, refinements of the Riemann Mapping Theorem also give very enlightening analytic information. The relevant analytic theory is the theory of prime ends. There is a good exposition of the theory in John B. Conway's readily available textbook, [6, Chapter 14, Sections 1-5]. It follows from the local connectivity of $M$ (applying Theorem 2.8(1)) that the impressions of the prime ends in $U$ are all singletons. By the theory of prime ends, the Riemann mapping from $\operatorname{int}\left(\mathbb{B}^{2}\right)$ onto $U$ extends continuously to the boundary.

Since the $\operatorname{arc} A$ is free, the prime ends at $A$ correspond exactly to the points of $A$ so that the map is one-to-one over $A$. 
Since the prime ends of $\operatorname{int}\left(\mathbb{B}^{2}\right)$ are singletons, the sets $f^{-1} f(p)$ are totally disconnected.

Proof of Theorem 1.2. Suppose that $M$ is a locally connected continuum in $\mathbb{S}^{2}$. If $M=\mathbb{S}^{2}$, then $M$ is already codiscrete. Otherwise, let $U_{1}, U_{2}, \ldots$ denote the complementary domains of $M$ in $\mathbb{S}^{2}$. By Theorem 2.8, the components of $\mathbb{S}^{2} \backslash M$ form a null sequence. By Theorem 2.9, there is for each $i$ a continuous surjection $f_{i}: \mathbb{B}^{2} \rightarrow \operatorname{cl}\left(U_{i}\right)$ that takes $\mathbb{S}^{1}$ onto the boundary of $U_{i}$ and takes the interior of $\mathbb{B}^{2}$ homeomorphically onto $U_{i}$. Let $p_{i}=f_{i}(0)$. Then the set $D=\left\{p_{1}, p_{2}, \ldots\right\}$ is obviously discrete. The set $\operatorname{cl}\left(U_{i}\right) \backslash\left\{p_{i}\right\}$ can obviously be deformed into the boundary of $U_{i}$ by pushing points away from $p_{i}$ along the images under $f_{i}$ of radii in $\mathbb{B}^{2}$. These deformations can be combined to deform all of $X=\mathbb{S}^{2} \backslash D$ onto $M$ since the $U_{i}$ form a null sequence. We conclude that $M$ is homotopy equivalent to the codiscrete set $X=\mathbb{S}^{2} \backslash D$.

Conversely, if $X$ is codiscrete, then we may take, about the points $p$ of $D(X)$, small disjoint round disks $d(p)$. The continuum $M=\mathbb{S}^{2} \backslash \bigcup_{p} \operatorname{int}(d(p))$ is a Peano continuum to which $X$ can be deformed by a strong deformation retraction.

This completes the proof of Theorem 1.2.

3. Peano domains. We may think of the Characterization Theorem 1.1 as a substantial generalization of the Filling Theorem, Theorem 2.9. We shall need an intermediate generalization of Theorem 2.9 that deals with compact sets that act much like Peano continua but are not necessarily connected. We call the complementary domains of such compacta Peano domains. We shall deal with the compacta themselves by joining them together by arcs so as to form a Peano continuum.

Definition 3.1. A connected open subset $U$ of $\mathbb{S}^{2}$ is called a Peano domain if its nondegenerate boundary components form a null sequence of Peano continua. (Note that there may be uncountably many additional components that are single points.)

THEOREM 3.2. Suppose that $U$ is a connected open subset of the 2sphere $\mathbb{S}^{2}$. Then the following three conditions are equivalent:

(1) The open set $U$ is a Peano domain.

(2) For each disk $D$ in $\mathbb{S}^{2}$, the components of $U \cap D$ form a null sequence.

(3) There is a continuous surjection $f: \mathbb{B}^{2} \rightarrow \operatorname{cl}(U)$ such that $f\left(\mathbb{S}^{1}\right) \supset$ $\partial(U)$ and $f \mid \operatorname{int}\left(\mathbb{B}^{2}\right)$ is a homeomorphism onto its image, which necessarily lies in $U$.

REMARK. Note that (1) generalizes the notion of local connectedness. Note that (2) generalizes characterization (1) of local connectedness in The- 
orem 2.8; the reader can reformulate (2) in each of the ways suggested by Theorem 2.8. Note that (3) generalizes Theorem 2.9. Note that, in the proof, we can assume that the map $f$ is 1-1 over given free boundary arcs of $U$ because the same is true in Theorem 2.9.

Proof. Assume (1), so that $U$ is a Peano domain. Assume that (2) is not satisfied, so that there is a disk $D$ in $\mathbb{S}^{2}$ such that the components of $U \cap D$ do not form a null sequence. Then some sequence $U_{1}, U_{2}, \ldots$ of components converges to a nondegenerate continuum $M$. The continuum $M$ must be a subset of a boundary component of $U$. We may assume that the components $U_{1}, U_{2}, \ldots$ are separated from each other by large boundary components of $U$. (Refer to the Brouwer Property II and to Corollary 2.2.) There are only finitely many large boundary components of $U$. Hence infinitely many of the separators must come from the same boundary component. It follows that the limit, namely $M$, is also in the same boundary component. But this boundary component is not locally connected at the points of $M$, a contradiction. We conclude that (2) is satisfied so that (1) implies (2).

Assume that (2) is satisfied but (1) is not. Then either there is a component of $\partial(U)$ that is not locally connected, or there exist infinitely many components of $\partial(U)$ having diameter $\geq \varepsilon$, for some fixed $\varepsilon>0$. In either case, taking a convergent sequence of large components or a limit continuum from a single component that is not locally connected (Theorem 2.8), we find the existence of an annulus $A$ in $\mathbb{S}^{2}$ and components $X_{1}, X_{2}, \ldots$ of $\partial(U) \cap A$, each of which intersects both components of $\partial(A)$. These components of $\partial(U) \cap A$ must be separated by large components of $A \cap U$. If we remove a slice from one of these large separating components, we obtain a disk $D$ that is crossed by infinitely many large components of $U \cap D$, which contradicts (2). Therefore (2) implies (1).

Assume that (3) is satisfied, so that there is a continuous surjection $f$ : $\mathbb{B}^{2} \rightarrow \operatorname{cl}(U)$ such that $f\left(\mathbb{S}^{1}\right) \supset \partial(U)$ and $f \mid \operatorname{int}\left(\mathbb{B}^{2}\right)$ is a homeomorphism onto its image. Assume that (1) is not satisfied, so that either there is a component of $\partial(U)$ that is not locally connected, or there exist infinitely many components of $\partial(U)$ each having diameter greater than some fixed positive number $\varepsilon$. In either case, we find by taking limits (Theorem 2.8) that there is an annulus $A$ in $\mathbb{S}^{2}$ and components $X_{1}, X_{2}, \ldots$ of $\partial(U) \cap A$, each of which intersects both components of $\partial(A)$. We may assume that $X_{1}, X_{2}, \ldots$ converges to a continuum $X_{0}$ joining both components of $\partial(A)$. We may assume that $X_{i-1} \cup X_{i+1}$ separates $X_{i}$ from $X_{0}$ in $A$, for $i=2,3, \ldots$.

Pick $p_{i} \in X_{i} \cap \operatorname{int}(A)$ for $i \geq 0$ such that $p_{i} \rightarrow p_{0}$. Let $q_{0}, q_{1}, q_{2}, \ldots \in \mathbb{S}^{1}$ be points such that $f\left(q_{i}\right)=p_{i}$. Let $B_{i}$ be the straight-line segment in $\mathbb{B}^{2}$ joining $q_{0}$ to $q_{i}$. We may assume that the $\operatorname{arcs} B_{i}$ converge to an arc or a point $B$ in $\mathbb{B}^{2}$. We shall obtain a contradiction as follows. 
The image $f\left(B_{i}\right)$ joins $X_{i}$ to $X_{0}$. It misses $X_{i-1} \cup X_{i+1} \subset \partial(U)$ since $f\left(q_{i}\right) \in X_{i}, f\left(q_{0}\right) \in X_{0}$, and $f\left(\operatorname{int}\left(B_{i}\right)\right) \subset U$. Hence, if we traverse $B_{i}$ from $q_{i}$ toward $q_{0}$, there exists a first point $b_{i} \in B_{i}$ such that $f\left(b_{i}\right) \in \partial(A)$. We may assume that $b_{i} \rightarrow b_{0} \in \mathbb{B}^{2}$ and $f\left(b_{i}\right) \rightarrow f\left(b_{0}\right) \in \partial(A)$. Since $f\left(b_{i}\right)$ is separated from $X_{0}$ by $X_{i-1} \cup X_{i+1}$ in $A$ and since $X_{i} \rightarrow X_{0}$, we may conclude that $f\left(b_{0}\right) \in X_{0} \cap \partial(A)$. Hence $b_{0} \in \mathbb{S}^{1} \backslash\left\{q_{0}\right\}$. But $b_{0}$ must therefore be an endpoint of $B$ distinct from $q_{0}$ and must therefore be the limit of the points $q_{i}$. We find that $f\left(q_{i}\right) \rightarrow p_{0} \in \operatorname{int}(A)$ and $f\left(q_{i}\right) \rightarrow f\left(b_{0}\right) \in \partial(A)$, a contradiction.

We conclude that (3) implies (1).

It remains to prove that (1) implies (3). This is by far the hardest of the implications. It is a generalization of the rather deep Theorem 2.9, and we shall reduce it to that theorem. We shall also make use of the wonderful R. L. Moore Decomposition Theorem 2.6.

Our plan is to connect $\partial(U)$ by deleting from $U$ a null sequence $A_{1}, A_{2}, \ldots$ of arcs to form a new connected open set $V=U \backslash \bigcup_{i} A_{i}$ whose boundary $\partial(V)=\partial(U) \cup \bigcup_{i} A_{i}$ is a locally connected continuum. Then we simply apply Theorem 2.9 .

For convenience, we wish to modify the closure of $U$ so that each nondegenerate boundary component of $\partial(U)$ is a simple closed curve. We shall do so in such a way that the closure of the new $U$ collapses to the closure of the old $U$ and preserves the property of being a Peano domain. This convenient, yet inessential, modification simplifies the description of the structures that we need to build in proving that $U$ is a Peano domain.

We change the nondegenerate components $C$ of $\partial(U)$ into simple closed curves as follows.

We define $U_{C}$ to be the component of $\mathbb{S}^{2} \backslash C$ that contains $U$. Since $C$ is locally connected by (1), we may apply Theorem 2.9 to find a continuous surjection $g: \mathbb{B}^{2} \rightarrow C \cup U_{C}$ that takes $\mathbb{S}^{1}$ onto $C$ and takes $\operatorname{int}\left(\mathbb{B}^{2}\right)$ homeomorphically onto $U_{C}$. We require that the sets $g^{-1} g(p)$ be totally disconnected for each $p \in \mathbb{S}^{1}=\partial\left(\mathbb{B}^{2}\right)$. Radii in $\mathbb{B}^{2}$ define radial segments in $U_{C}$ whose endpoints may be identified at various points of $\partial\left(U_{C}\right)$.

We pull $U_{C}$ into itself along these radial segments so as to split the boundary identifications of $g \mid\left(\mathbb{S}^{1}=\partial\left(\mathbb{B}^{2}\right)\right)$ apart and make $\partial\left(U_{C}\right)$ a simple closed curve. Because the sets $g^{-1} g(p)$ are totally disconnected, it follows that the nondegenerate boundary components of the new $U$ also form a null sequence. We may thus assume that $\partial\left(U_{C}\right)$ is a simple closed curve.

We repeat the argument with each of the countably many nondegenerate boundary components in turn. Since each move can be made arbitrarily small, there is no problem in getting the sequence of moves to converge. The difficulty lies in getting all of the appropriate properties to be preserved in the limit. It is possible to deal with that difficulty exactly as one does in the proof of the Baire Category Theorem, in the embedding of $n$-dimensional com- 
pacta in Euclidean $2 n+1$-dimensional space (see, for example, the proof of [1, Chapter VI, Theorem 1.52]), or in forcing a sequence of homeomorphisms to converge to a homeomorphism: namely, the desired properties can be encoded in countably many open conditions in function space, and each successive change can be made so small that more and more of the desired conditions are satisfied and preserved in the limit.

We may thus repeat the argument infinitely often to conclude that we lose no generality in assuming that each nondegenerate component is a simple closed curve. That is, $U$ is the complement of a null sequence of disks $D_{1}, D_{2}, \ldots$ and a 0 -dimensional set $D$, the union of $D_{1}, D_{2}, \ldots$, and $D$ being closed.

We shall string the components of $\partial(U)$ together by a null sequence of arcs that run through $U$. These arcs will be built by approximation. At every stage, the arcs together with certain larger and larger collections of the disks $D_{j}$ will form a contractible set. The additions at each stage will be smaller and smaller "feelers". The feelers will be buffered from one another so that, in the limit, they cannot grow back together. It follows that the limit continuum cannot separate $\mathbb{S}^{2}$. Certain limit points in $\partial(U)$ will be attached only in the limit. The structures defined make it clear that the limiting continuum can be expressed as a union of finitely many arbitrarily small connected sets so that the final continuum is a single Peano continuum. Here is the process:

It may help the reader to imagine that the closure of $U$ lies in the unit square $[0,1] \times[0,1]$, that one of the disks $D_{1}$ is the complement of this square in the compactified plane $\mathbb{S}^{2}=\mathbb{R}^{2} \cup\{\infty\}$, and that the other disks $D_{2}, D_{3}, \ldots$ are some subcollection of the disks whose interiors are removed in forming the standard Sierpiński curve. This is permissible by a theorem of G. T. Whyburn [18]. However, this normalization is a conceptual aid only since we have to enrich the Whyburn argument a bit. We explain the mild complications that arise in our setting in the next paragraph.

We wish to construct a nice sequence of cellulations of the 2-sphere that respect the boundary components of $U$. If, for example, we wish to concentrate on some particular finite set $S$ of the large disks $D_{i}$, we may form an upper semicontinuous decomposition of $\mathbb{S}^{2}$ by declaring the other $D_{i}$ 's that miss $S$ to be the nondegenerate elements of the decomposition. By R. L. Moore's Decomposition Theorem 2.6, the quotient space is the 2 -sphere $\mathbb{S}^{2}$. The (homeomorphic) image of $U$ in this new copy of $\mathbb{S}^{2}$ will have, as complement, the (images of the) elements of $S$ and a 0 -dimensional set that is locally closed away from $S$. It is then an easy matter to cellulate $\mathbb{S}^{2}$ so that the elements of $S$ cover a subcomplex and the remainder of the 1-skeleton misses $\partial(U)$ entirely. This adjustment of the 1-skeleton is the central part of the Whyburn argument [18]. Whyburn only has to have his 
1-skeleton miss a countable set. We need the 1-skeleton to miss a countable family of closed, 0-dimensional sets. But since the complement of a closed 0 -dimensional set is locally path connected, our requirements are no harder to meet than his.

As a consequence, we find that there is a sequence $S_{1}, S_{2}, \ldots$ of arbitrarily fine cellulations of $\mathbb{S}^{2}, S_{i+1}$ subdividing $S_{i}$, such that, for each $i$, the following conditions are satisfied:

(i) Two 2-cells of $S_{i}$ that intersect intersect in an arc.

(ii) The 1-skeleton of $S_{i}$ misses all of the 0-dimensional part $D$ of $\partial(U)$.

(iii) For all $j$, the 1-skeleton of $S_{i}$ either misses the disk $D_{j}$ or contains $\partial\left(D_{j}\right)$. Consequently, $S_{i}$ has a distinguished finite subcollection of disks $D_{j}$ that are precisely equal to unions of 2-cells of $S_{i}$. All other disks $D_{k}$ will lie in the interiors of 2-cells of $S_{i}$.

(iv) If a 2-cell $C$ of $S_{i}$ has a boundary point in some $\partial\left(D_{j}\right)$, with $\operatorname{int}(C)$ $\not \subset D_{j}$, then $\partial(C) \cap \bigcup_{k} D_{k}$ is an arc in $\partial\left(D_{j}\right)$.

It is necessary to distinguish four types of 2-cells in the cellulation $S_{i}$ :

A 2-cell $C$ of $S_{i}$ is of type 0 if it lies entirely in $U$.

A 2-cell $C$ is of type 1 if it lies entirely in the complement of $U$, hence lies in one of the distinguished disks $D_{j}$ of the cellulation $S_{i}$ (see (iii) above).

A 2-cell $C$ is of type 2 if it intersects both $U$ and the complement of $U$, but its boundary lies entirely in $U$.

A 2-cell $C$ is of type 3 if its boundary intersects both $U$ and the complement of $U$. Condition (iv) above implies that a 2-cell $C$ of type 3 has boundary that intersects precisely one disk $D_{j}$, that $D_{j}$ is one of the distinguished disks of $S_{i}$, and the intersection is a boundary arc of each.

We shall essentially ignore the 2-cells of type 0 . We shall deal with the disks of type 1 only implicitly by considering instead their unions that give the distinguished disks $D_{j}$ of the cellulation $S_{i}$ (see (iii) above). Cells of type 2 will be joined to these distinguished disks by arcs in $U$. Cells of type 3 will be joined to these distinguished disks by their intersecting boundary arcs.

It will be convenient to use the notation $C^{*}$ for the union of the elements of a collection $C$ of sets.

Let $\mathcal{D}_{1}$ denote the collection of $D_{j}$ 's that are distinguished in the cellulation $S_{1}$. Then $\mathcal{D}_{1}^{*}=\bigcup\left\{D \in \mathcal{D}_{1}\right\}$. We may assume $D_{1} \in \mathcal{D}_{1}$. We may pick a collection $\mathcal{A}_{1}$ of arcs from the 1-skeleton $S_{1}^{(1)}$ of $S_{1}$ that irreducibly joins together these distinguished disks $D_{j} \in \mathcal{D}_{1}$, so that the union $\mathcal{C}_{1}=\mathcal{D}_{1}^{*} \cup \mathcal{A}_{1}^{*}$ of disks and arcs is contractible. Without destroying the contractibility of the set $\mathcal{C}_{1}$, we add additional arcs from the 1 -skeleton to the collection $\mathcal{A}_{1}$ if necessary so that every cell of type 2 intersects one of the arcs of $\mathcal{A}_{1}$.

All of the cells of $S_{1}$ of type 0 will be ignored from now on. All of the cells of $S_{1}$ of type 1 are contained in the contractible topological polyhedron $\mathcal{C}_{1}$. 
As we proceed to the induction, we will attach "feelers" to $\mathcal{C}_{1}$. There will be one feeler in each cell $C$ of type 2 and it will be attached to $\mathcal{C}_{1}$ at a single, arbitrarily chosen point of the intersection of $C \cap \mathcal{C}_{1}$. We call that point the attaching point. In each cell $C$ of type 3 , there may be finitely many or a null sequence of feelers, but each will be attached to $\mathcal{C}_{1}$ at some point of the boundary arc of $C$ that lies in a distinguished disk. We call that arc the attaching arc. There may certainly be other points of $\partial(C)$ that lie in $\mathcal{C}_{1}$, but none of these lies in $\partial(U)$. Hence, for cells $C$ of types 2 and 3, the complement in $\partial(C)$ of the attaching point or attaching arc can serve as a buffer never to be approached or crossed in the construction. It is these buffers that make it easy to see that the limit continuum is contractible and locally connected.

We proceed by induction. We assume that we have constructed contractible sets $\mathcal{C}_{1} \subset \cdots \subset \mathcal{C}_{i}$ that lie except for distinguished disks of $S_{1}, S_{2}$, $\ldots, S_{i}$ in the 1 -skeletons of the cellulations. We may impose one additional condition on the cellulation $S_{i+1}$ :

(v) For each cell $C$ of $S_{i}$ that has type 2 or 3, that part of the 1-skeleton of $S_{i+1}$ that lies in the interior of $C$, taken together with the attaching point (type 2) or attaching arc (type 3 ), is connected.

All of the action in creating $\mathcal{C}_{i+1}$ takes place in the individual cells $C$ of $S_{i}$ of type 2 and 3 . Exactly as in the construction of $\mathcal{C}_{1}$, we may pick a collection of arcs $\mathcal{A}_{i+1}(C)$ from that part of the 1-skeleton of $S_{i+1}$ that lies in the interior of $C$, taken together with the attaching point (type 2) or attaching arc (type 3 ), that irreducibly joins together the attaching set of $C$, the distinguished disks $D_{j} \in \mathcal{D}_{i+1}$ in $C$, and the cells of $S_{i+1}$ of type 2 in $C$. All of these new distinguished disks and all of these new arcs can be added to $\mathcal{C}_{i}$ to form a new contractible set $\mathcal{C}_{i+1}$. We denote the entire union $\bigcup_{C} \mathcal{A}_{i+1}(C)$ of $\operatorname{arcs}$ as $\mathcal{A}_{i+1}$.

For each of the new cells of types 2 and 3 , we choose an attaching point or arc as before.

We leave it to the reader to verify that $M=\left(\mathbb{S}^{2} \backslash U\right) \cup \bigcup_{i} A_{i}$ is a single locally connected continuum with a single complementary domain $V=$ $U \backslash \bigcup_{i} A_{i}$.

By Theorem 2.9, there is a map $f: \mathbb{B}^{2} \rightarrow \operatorname{cl}(V)$ from the 2-disk $\mathbb{B}^{2}$ onto the closure of the domain $V$ that takes $\operatorname{int}\left(B^{2}\right)$ homeomorphically onto $V$ and takes $\mathbb{S}^{1}=\partial\left(\mathbb{B}^{2}\right)$ continuously onto $\partial(V)$. The same map establishes condition (3) of Theorem 3.2.

This completes the proof that (1) implies (3). Thus all three conditions of Theorem 3.2 are equivalent, as claimed. The proof of Theorem 3.2 is therefore complete.

Our final theorem of this section shows how to push a Peano domain onto its boundary together with a 1-dimensional set provided the domain is 
punctured on a nonempty discrete set. This easy theorem will be needed as the last step in the proof of Theorem 1.1.

Theorem 3.3. Suppose that $U$ is a Peano domain in $\mathbb{S}^{2}$ and that $C$ is a nonempty countable or finite subset of $U$ that has no limit points in $U$. Then $\operatorname{cl}(U) \backslash C$ can be retracted by a strong deformation retraction onto a 1-dimensional compactum that contains $\partial(U)$.

Proof. By Theorem 3.2, we know that there is a continuous surjection $f: \mathbb{B}^{2} \rightarrow \operatorname{cl}(U)$ such that $f\left(\mathbb{S}^{1}\right) \supset \partial(U)$ and $f \mid \operatorname{int}\left(\mathbb{B}^{2}\right)$ is a homeomorphism onto its image.

By invariance of domain, $f \mid \operatorname{int}\left(\mathbb{B}^{2}\right)$ is an open map. It follows that $f\left(\operatorname{int}\left(\mathbb{B}^{2}\right)\right)$ is disjoint from $f\left(\partial\left(\mathbb{B}^{2}\right)\right)=f\left(\mathbb{S}^{1}\right)$, for otherwise points of $\operatorname{int}\left(\mathbb{B}^{2}\right)$ near $\mathbb{S}^{1}$ would map to points already occupied by other points of $f\left(\operatorname{int}\left(\mathbb{B}^{2}\right)\right)$.

Since $f\left(\operatorname{int}\left(\mathbb{B}^{2}\right)\right)$ is dense in $f\left(\mathbb{B}^{2}\right)=\operatorname{cl}(U)$ and disjoint from $f\left(\mathbb{S}^{1}\right), f\left(\mathbb{S}^{1}\right)$ must be 1-dimensional. Hence it is an easy exercise to show that we may modify $f$ slightly over $U$ so that $f\left(\mathbb{S}^{1}\right)$ misses $C$. We may further modify $f$ so that $f$ maps the origin $0 \in \mathbb{B}^{2}$ to a point of $C$ and so that all other points of $C$ have preimages on different radii of $\mathbb{B}^{2}$. Let $f^{-1}(C)=\left\{c_{0}=0, c_{1}, c_{2}, c_{3}, \ldots\right\}$. Let $A_{1}, A_{2}, \ldots$ be the radial arcs beginning at $c_{1}, c_{2}, \ldots$, respectively, and ending on $\mathbb{S}^{1}=\partial\left(\mathbb{B}^{2}\right)$. Let $D_{1}, D_{2}, \ldots$ be disjoint round disks $\operatorname{in} \operatorname{int}\left(\mathbb{B}^{2}\right) \backslash\{0\}$ centered at $c_{1}, c_{2}, \ldots$, respectively, such that the only $A_{j}$ intersected by $D_{i}$ is $A_{i}$. Let $V=\operatorname{int}\left(\mathbb{B}^{2}\right) \backslash\left[\bigcup_{i} A_{i} \cup \bigcup_{i} D_{i}\right]$. Then $\mathbb{B}^{2} \backslash f^{-1}(C)$ can obviously be retracted by a strong deformation retraction onto the 1-dimensional set $\partial(V)$. Hence $f\left(\mathbb{B}^{2}\right) \backslash C=\operatorname{cl}(U) \backslash C$ can be retracted by a strong deformation retraction onto the 1 -dimensional set $f(\partial(V))$.

\section{The necessity of conditions (1) and (2) in Theorem 1.1. We} assume that $X$ is a codiscrete set that is homotopy equivalent to a metric 1-dimensional set $Y$. Let $f: X \rightarrow Y$ and $g: Y \rightarrow X$ be homotopy inverses.

We isolate the three key technical constructions as lemmas. Each of these is standard and well-known. We omit the proofs.

Lemma 4.1 (Dimension Lemma). If $g: Z^{\prime} \rightarrow Z$ is any map from a 1-dimensional compactum $Z^{\prime}$ into the closure $Z$ of an open subset $U$ of $\mathbb{S}^{2}$, then $g$ is homotopic, by a homotopy which only moves points in $U$ to a map $g^{\prime}: Z^{\prime} \rightarrow Z$ such that $g^{\prime}\left(Z^{\prime}\right) \cap U$ is 1-dimensional. (The key ideas are explained, for example, in [17, Exercises for Chapter 3, Sections G and H].)

Lemma 4.2 (Homotopy Lemma).

(i) Let $C \subset \mathbb{S}^{2}$ be closed, and let $H: C \times[0,1] \rightarrow \mathbb{S}^{2}$ denote a deformation of $C$ that begins at the identity (that is, $H(c, 0)=c$ for all $c \in C)$. Then $H$ can be extended to a deformation $H^{\prime}: \mathbb{S}^{2} \times[0,1] \rightarrow \mathbb{S}^{2}$ that also begins at the identity. 
(ii) If $H$ moves no point as far as $\varepsilon>0$, then we may require that $H^{\prime}$ have the same property.

(iii) If $N$ is an open set in $\mathbb{S}^{2}$ containing the points of $\partial(C)$ that are moved by the deformation $H$, then we may require that $N$ contain the points of $\mathbb{S}^{2} \backslash C$ moved by $H^{\prime}$. (See [16, Section 62, Lemma 62.1 and Exercise 3].)

Lemma 4.3 (Annulus Lemma). Suppose condition (2) of Theorem 1.1 fails. Then there are an annulus $R^{\prime}$ in $\mathbb{S}^{2}$ and components $U_{1}^{\prime}, U_{2}^{\prime}, \ldots$ of $R^{\prime} \backslash B(X)$ such that each $U_{j}^{\prime}$ intersects both boundary components of $R^{\prime}$ and misses the set $D(X)$. (See Theorem 2.8 and its proof.)

The three lemmas imply necessity of conditions (1) and (2) as follows: By precomposing the homotopy equivalence $f$ with a deformation retraction onto a compact subset of $X$, we may assume that the image $f(X)$ is a 1 -dimensional continuum $Z^{\prime}$. By Dimension Lemma 4.1, we may assume that $g \circ f(X) \backslash B(X)$ is 1-dimensional. Let $G: X \times[0,1] \rightarrow X$ be a homotopy that begins with the identity on $X$ and ends with $g \circ f$. By Theorem 2.3, we see that $G(x, t)=x$ for each $x \in B(X)$.

Assume that condition (1) of the hypothesis of Theorem 1.1 fails, so that some component $U$ of $\mathbb{S}^{2} \backslash B(X)$ contains no point of $D(X)$. Hence $U \subset X$. Let $H: \operatorname{cl}(U) \times[0,1] \rightarrow \mathbb{S}^{2}$ denote the restriction of $G$ to $\operatorname{cl}(U) \times[0,1]$. Since $H$ fixes $\partial(U) \subset B(X)$, we may extend $H$ to a deformation $H^{\prime}$ of $\mathbb{S}^{2}$ that fixes $\mathbb{S}^{2} \backslash U$ pointwise. Since $H^{\prime}\left(\mathbb{S}^{2} \times\{1\}\right) \cap U \subset G\left(\mathbb{S}^{2} \times\{1\}\right) \cap U$ is 1-dimensional, we see that $H^{\prime}$ deforms $\mathbb{S}^{2}$ into a proper subset of itself, which is impossible. Hence condition (1) must be satisfied.

Assume that condition (2) of the hypothesis of Theorem 1.1 fails. Then, by Annulus Lemma 4.3, there are an annulus $R^{\prime}$ in $\mathbb{S}^{2}$ and components $U_{1}^{\prime}, U_{2}^{\prime}, \ldots$ of $R^{\prime} \backslash B(X)$ such that each $U_{j}^{\prime}$ intersects both boundary components of $R^{\prime}$ and fails to intersect the set $D(X)$.

By passing to a subsequence, we may assume that the components $U_{1}^{\prime}, U_{2}^{\prime}$, $U_{3}^{\prime}, \ldots$ converge to a continuum $A$ that joins the two boundary components of $R^{\prime}$. Since the components $U_{j}^{\prime}$ are separated by $B(X)$, it follows that $A \subset$ $B(X)$. Let $D$ be a small disk in $\operatorname{int}\left(R^{\prime}\right)$ centered at some point of $A$. Since the deformation $G$ constructed above moves no point of $B(X)$, there is a neighborhood $N$ of $A$ in $X$, no point of which is moved by $G$ as far as $1 / 2$, the distance from $\partial\left(R^{\prime}\right)$ to $D$. We choose $j$ so large that $\operatorname{cl}\left(U_{j}^{\prime}\right) \subset N$ and $U_{j}^{\prime} \cap \operatorname{int}(D) \neq \emptyset$. Since no point of $D(X)$ lies in $U_{j}^{\prime}$, all of $\operatorname{cl}\left(U_{j}^{\prime}\right)$ lies in $X$.

We let $H: \operatorname{cl}\left(U_{j}^{\prime}\right) \times[0,1] \rightarrow \mathbb{S}^{2}$ be the restriction of $G$ to $\operatorname{cl}\left(U_{j}^{\prime}\right) \times[0,1]$. By Homotopy Lemma 4.2(i), there is a deformation $H^{\prime}: \mathbb{S}^{2} \times[0,1] \rightarrow \mathbb{S}^{2}$ that extends $H$. Since $\partial\left(U_{j}^{\prime}\right) \cap \operatorname{int}\left(R^{\prime}\right) \subset B(X), H$ does not move the points of $\partial\left(U_{j}^{\prime}\right) \cap \operatorname{int}\left(R^{\prime}\right)$. Hence, by Homotopy Lemma 4.2(iii), we may require that $H^{\prime} \mid\left[\mathbb{S}^{2} \backslash \operatorname{cl}\left(U_{j}^{\prime}\right)\right] \times[0,1]$ moves points only near $\partial(R) \cap \operatorname{cl}\left(U_{j}^{\prime}\right)$, a set that 
contains the points moved by $H \mid \partial\left(U_{j}^{\prime}\right) \times[0,1]$. By Homotopy Lemma 4.2(ii), we may require that no points of $\mathbb{S}^{2} \backslash U_{j}^{\prime}$ be carried into $D \cap U_{j}^{\prime}$. Hence $H^{\prime}$ is a homotopy of $\mathbb{S}^{2}$ that takes $\mathbb{S}^{2}$ to a proper subset of itself, an impossibility. Hence condition (2) of Theorem 1.1 is also satisfied.

\section{The sufficiency of conditions (1) and (2) in Characterization} Theorem 1.1. We assume conditions (1) and (2) of Characterization Theorem 1.1. That is, the open set $U_{0}=\mathbb{S}^{2} \backslash B(X)$ satisfies the following two conditions:

(1) Each component of $U_{0}$ contains a point of $D(X)$.

(2) If $D$ is any disk in $\mathbb{S}^{2}$, then the components of $U_{0} \cap D$ that contain no point of $D(X)$ form a null sequence.

Our goal is to show that $X$ is homotopy equivalent to a 1-dimensional set.

Notice that properties (1) and (2) make no explicit mention of the bad set $B(X)$ and are simply properties that an open subset of $\mathbb{S}^{2}$ may or may not have. This is an important observation, because our proof that $X$ is homotopy equivalent to a 1-dimensional set will involve a complicated induction that will involve a null sequence $U_{0}, U_{1}, U_{2}, \ldots$ of open sets, each of which satisfies properties (1) and (2).

It will also be convenient to adopt the following terminology: we say that set is punctured if it contains a point of $D(X)$. Otherwise, we say that it is unpunctured.

We first have to deal with the trivial case where $B(X)=\emptyset$. If $B(X)=\emptyset$, then the single component $\mathbb{S}^{2}=\mathbb{S}^{2} \backslash B(X)$ must contain a point of $D(X)$ by (1). Thus there must be at least one point of $D(X)$ and at most finitely many. Hence $X$ is clearly homotopy equivalent to a point or bouquet of circles.

From now on, we may assume that the set $D(X)$ is infinite and the set $B(X)$ is nonempty. Since $D(X)$ is countable, we may list the points $p_{0}, p_{1}, p_{2}, \ldots$ of $D(X)$. We need to show that $X$ is homotopy equivalent to a 1-dimensional set. We shall do this by constructing a null sequence $U_{0}, U_{1}, U_{2}, \ldots$ of disjoint Peano domains such that, for each $i, p_{i} \in U_{i}$, and such that the union $\bigcup_{i} U_{i}$ is dense in $\mathbb{S}^{2}$. Each set $\operatorname{cl}\left(U_{i}\right) \backslash\left\{p_{i}\right\}$ can be deformed onto a 1-dimensional set that contains its boundary by Theorem 3.3. Since these sets form a null sequence, the deformations can be combined to give a deformation that takes $X$ onto the union of $\mathbb{S}^{2} \backslash \bigcup_{i} U_{i}$ and 1-dimensional compacta that contain the sets $\partial\left(U_{i}\right)$. Each of these sets is a compact 1dimensional set. Hence their (countable) union is 1-dimensional.

The domains $U_{i}$ are created by a long induction. Each step of the induction constructs a null sequence of Peano domains. At step 0 of the induction, an individual domain can have diameter as large as the diameter of $\mathbb{S}^{2}$. There- 
after, however, we may restrict the maximum diameter of a Peano domain at step $i$ to be bounded by $1 / i$. Hence the union of this countable collection of null sequences is also a null sequence.

We consider $\mathbb{S}^{2}$ as $\mathbb{R}^{2} \cup\{\infty\}$. We may assume that $p_{0}=\infty \in D(X)$. By scaling and translating $\mathbb{R}^{2}$, we may assume that $[D(X) \backslash\{\infty\}] \cup B(X)$ lies in the interior of the closed unit square $S=[0,1] \times[0,1]$.

We now begin the construction of our first null sequence of Peano domains. We outline the strategy. The reader who digests this strategy will be able to avoid getting lost in the details. We are trying to fill the open set $U_{0}=\mathbb{S}^{2} \backslash B(X)$ with small Peano domains, more precisely a null sequence of Peano domains, that are punctured (contain points of $D(X)$ ). We therefore cover $U_{0}$ with a fine grid to divide it into small pieces. What happens then is reminiscent of the children's story, "Fortunately" ([5]). Fortunately, some of these small pieces will be punctured. Unfortunately, some will be unpunctured. Fortunately, the unpunctured pieces form a null sequence by hypothesis (2); unfortunately, however, they must be attached to adjacent pieces that are punctured and, unfortunately, the adjacent punctured pieces need not form a null sequence. Fortunately, we can carve out of the adjacent punctured pieces a null sequence of smaller punctured pieces to which we can attach the unpunctured pieces. Unfortunately, the process of carving out small punctured pieces creates new unpunctured pieces. Fortunately, the new unpunctured pieces form a null sequence that we can attach to the null sequence of punctured pieces. Unfortunately, the carving out of small punctured pieces creates new, as yet unattached, punctured pieces that need not form a null sequence. Fortunately, the unattached punctured pieces are uniformly small and, together, form a new open set $U_{1}$ that satisfies hypotheses (1) and (2). We can then undertake the inductive step with a new open set whose pieces are smaller than at the previous stage. Here are the details.

STEP 1: Creating small pieces. We impose a square grid on $S$ consisting of a large square formed from small constituent closed squares. Since the set $D(X)$ is countable, we lose no generality in assuming that the edges of the grid miss $D(X)$. The grid divides the open set $U_{0}=\mathbb{S}^{2} \backslash B(X)$ into many components. We call the collection of such components $\mathcal{C}_{0}$. More precisely:

(i) The set $\mathbb{S}^{2} \backslash \operatorname{int}(S)$ is an element of $\mathcal{C}_{0}$.

(ii) If $T$ is any small, closed, constituent square of the grid, then each component of $T \backslash B(X)$ is also an element of $\mathcal{C}_{0}$.

Note that the elements of $\mathcal{C}_{0}$ are not in general disjoint since they can intersect along the edges of the grid.

STEP 2: Collecting the unpunctured pieces into a null sequence of small sets. Let $\mathcal{C}_{0}^{\prime}$ denote the subcollection of $\mathcal{C}_{0}$ consisting of those elements whose 
interiors are unpunctured. We take the union $\cup \mathcal{C}_{0}^{\prime}$ of the elements of $C_{0}^{\prime}$ and claim two things:

(iii) The components of $\bigcup \mathcal{C}_{0}^{\prime}$ form a null sequence.

(iv) Each component of $\bigcup \mathcal{C}_{0}^{\prime}$ shares points of an edge with an element of $\mathcal{C}_{0}$ whose interior is punctured.

Proof of (iii). We apply here the fundamental principle of convergence of continua from Theorems 2.4 and 2.5. The argument could be repeated almost verbatim perhaps four more times in the course of Section 4. Often we will have to consider two cases, depending on whether the limit continuum contains a point in the interior of a constituent square of the superimposed grid or does not. We will not always repeat the details after this first argument. Here are the details:

Suppose $\varepsilon>0$, and suppose that there exist components $Y_{1}, Y_{2}, \ldots$ of $\cup \mathcal{C}_{0}^{\prime}$, each of diameter $\geq \varepsilon$. We may assume that $Y_{i} \rightarrow Y$ in the sense of Theorems 2.4 and 2.5, where $Y$ is a continuum of diameter $\geq \varepsilon$.

Suppose first that $Y$ contains a point in the interior of some constituent square. Then a small annulus $A$ about that point in the interior of the constituent square intersects all but finitely many of the $Y_{i}$ in a component that crosses $A$ from one boundary component to the other, which easily gives a contradiction to hypothesis (2).

Suppose next that $Y$ lies in the 1-skeleton of the grid. Then it contains an interval of an edge of one of the small constituent squares. In this case, we may take an annulus $A$ that surrounds an interior point of the interval and intersects each of the two adjacent squares in a disk (half of an annulus). Again, all but finitely many of the $Y_{i}$ will intersect one of these two disks in a component that crosses the disk from one side to the opposite, which easily gives a contradiction to hypothesis (2).

This completes the proof of (iii).

Proof of $(i v)$. We may expand the elements of $\mathcal{C}_{0}$ slightly without introducing intersections between sets that did not already intersect; we thus obtain an open covering of $U_{0}$. Each component of $U_{0}$ is punctured, by hypothesis (1). In each component $V$, any two elements of $\mathcal{C}_{0}$, as expanded, that lie in $V$ are joined by a finite chain of such elements by a standard connectedness argument. A minimal such chain connects each element of $\mathcal{C}_{0}^{\prime}$ to an element of $\mathcal{C}_{0}$ that is punctured. Property (iv) follows.

STEP 3: Attaching the unpunctured pieces of Step 2 to a null sequence of punctured pieces. To each component $K$ of $\bigcup \mathcal{C}_{0}^{\prime}$ we assign a punctured element $L=L(K) \in \mathcal{C}_{0}$ that intersects $K$ along at least one edge. Such an element $L(K)$ exists by (iv) of Step 2. The elements $L$ thus chosen definitely need not form a null sequence, but we shall carve out from such elements 
$L$ a new null sequence of punctured domains to which we may attach the components $K$. Here is the argument:

For each component $K$, choose an open arc $A(K)$ along which $K$ is attached to $L(K)$. Choose a point $q(K) \in A(K)$. Enumerate these points as $q_{1}, q_{2}, \ldots$ Each $q_{i}$ belongs to a specific $K_{i}$, and arc $A_{i}$, and component $L_{i}=L\left(K_{i}\right)$.

Choose an arc $B_{1}$ in $L_{1}$ that joins $q_{1}$ to $D(X)$ irreducibly. We may require that $B_{1} \cap$ (1-skeleton of grid $)=q_{1}$ and that, for every arc $B$ having the same properties, $\operatorname{diam}\left(B_{1}\right) \leq 2 \operatorname{diam}(B)$.

Proceed inductively. Choose an arc $B_{k+1}$ in $L_{k+1}$ joining $q_{k+1}$ to $D(X) \cup$ $B_{1} \cup \cdots \cup B_{k}$ irreducibly. We may require that $B_{k+1} \cap(1$-skeleton of grid $)=$ $q_{k+1}$ and that, for every arc $B$ having the same properties, $\operatorname{diam}\left(B_{k+1}\right) \leq$ $2 \operatorname{diam}(B)$.

We make the following claims about the $\operatorname{arcs} B_{i}$ :

(v) The arcs $B_{1}, B_{2}, \ldots$ form a null sequence.

(vi) For every $\varepsilon>0$, there exists $k$ such that each component of $\mathcal{B}(k)=$ $B_{k+1} \cup B_{k+2} \cup \cdots$ has diameter less than $\varepsilon$.

[Note that (vi) implies (v). Properties (v) and (vi) are stated separately since $(\mathrm{v})$ is used in the proof of (vi).]

Proof of $(v)$. Suppose that $(\mathrm{v})$ is not satisfied. Then there is a subsequence $B_{i_{1}}, B_{i_{2}}, \ldots$ that converges to a nondegenerate continuum $B$. [This is our second application of the fundamental principle of Theorems 2.4 and 2.5.] We may assume that the $B_{i_{j}}$ all lie in the same small constituent square $T$ of the grid and that their initial endpoints $q_{i_{1}}, q_{i_{2}}, \ldots$ converge to a point $q \in \partial(T)$. Let $A$ be a small annulus about $q$ that intersects $T$ in a small disk $A^{\prime}$ of less than half of the diameter of $B$. All but finitely many of the arcs $B_{i_{j}}$ cross that disk $A^{\prime}$ in a large component $B_{i_{j}}^{\prime}$. By hypothesis (2), only finitely many large components of $A^{\prime} \cap U_{0}$ do not contain a point of $D(X)$. It follows easily that either some $B_{i_{j}}^{\prime}$ is in a component that contains a point of $D(X)$ or is in a component that contains another $B_{i_{k}}^{\prime}$, with $j>k$. In either case, the diameter of $B_{i_{j}}$ can be reduced more than half by shortcutting $B_{i_{j}}$ to $D(X)$ or to $B_{i_{k}}$, a contradiction. This completes the proof of $(\mathrm{v})$.

Proof of (vi). We shall make strong use of (v). Suppose there is an $\varepsilon>0$ such that each of the sets $\mathcal{B}(k)=B_{k+1} \cup B_{k+2} \cup \cdots$ contains a component $Y_{k}$ of diameter $\geq \varepsilon$. We may pick from $Y_{k}$ a subset $Y_{k}^{\prime}$ that is a finite chain $Y_{k}^{\prime}=B_{k_{1}} \cup \cdots \cup B_{k_{l}}$ of the arcs $B_{1}, B_{2}, \ldots$ and that has diameter $\geq \varepsilon / 2$. We may assume that the sets $Y_{k}^{\prime}$ are disjoint. Indeed, passing to a subsequence, we may certainly assume that different $Y_{k}^{\prime}$ 's involve different $B_{k_{j}}$ 's. Then, since the components of $\mathcal{B}(1)$ are trees, one $Y_{k}^{\prime}$ can intersect another only in one point. Hence, if one deletes the $\mathcal{B}_{k_{j}}$ intersecting the previous $Y_{k}^{\prime}$, one 
will still have at least one subchain of substantial size (approximately $\varepsilon / 4$ ) that is disjoint from the previous $Y_{k}^{\prime}$. Passing to an appropriate subsequence, we may certainly assume that the $Y_{k}^{\prime}$ all lie in the same small constituent square $T$. If $Y_{k}^{\prime}=B_{k_{1}} \cup \cdots \cup B_{k_{l}}$ with $k_{1}<\cdots<k_{l}$, then we call $q(k)=q_{k_{l}}$ the initial point of $Y_{k}^{\prime}$. We may assume that the initial points $q(k)$ converge to a point $q \in \partial(T)$. Let $A$ be a small annulus about $q$ that intersects $T$ in a small disk $A^{\prime}$. Then each $Y_{k}^{\prime}$ is a chain of small arcs crossing $A^{\prime}$ whose links $B_{k_{j}}$ all intersect $\partial(T)$. A $Y_{k}^{\prime}$ with small links, each intersecting $\partial(T)$, must hug $\partial(T)$ as it crosses $A^{\prime}$ and presents a barrier that cannot be avoided by another (disjoint) $Y_{k}^{\prime}$ that crosses $A^{\prime}$ in the same direction. It follows that there can be at most two such that are disjoint, each crossing $A^{\prime}$ along $\partial(T)$ in a different direction, a contradiction. This completes the proof of (vi).

From property (vi) it follows easily that each component $B$ of $B_{1} \cup B_{2} \cup \ldots$ is a tree that lies in a single small constituent square $T$, contains exactly one point of $D(X)$, and has, as its leaves (leaf = vertex lying on only one edge), special attaching points $q_{j}$ in corresponding attaching arcs $A_{j}$ of certain components $K_{j}$ of $\cup \mathcal{C}_{0}^{\prime}$. Furthermore, these trees $B$ form a null sequence of trees.

Each component of $\bigcup \mathcal{C}_{0}^{\prime}$ is attached to one of these trees at a leaf. We thicken each of these trees slightly and disjointly so that they still form a null sequence, still contain one point of $D(X)$ each, but now intersect the appropriate attaching arcs $A_{j}$ in neighborhoods $A_{j}^{\prime}$ of the attaching points $q_{j}$.

If $B$ is one of the tree components of $B_{1} \cup B_{2} \cup \cdots$, then we let $B^{\prime}$ denote its thickening. We let $B_{1}^{\prime}, B_{2}^{\prime}, \ldots$ denote the collection of thickened trees.

The interiors of the thickened trees $B_{j}^{\prime}$ are clearly Peano domains since it is an easy matter to construct a continuous surjection $f: \mathbb{B}^{2} \rightarrow \operatorname{cl}\left(B_{j}^{\prime}\right)$ that takes $\operatorname{int}\left(\mathbb{B}^{2}\right)$ homeomorphically onto int $\left(B_{j}^{\prime}\right)$. These Peano domains will form the cores of the Peano domains that we are attempting to construct in this stage of the induction. To them, we must attach the components $K_{j}$ that we have described above and also certain sets that we will describe in the next step.

STEP 4: Attaching the unpunctured components created by removing the thickened trees of Step 3. When we remove the thickened trees $B_{j}^{\prime}$ from the components $L=L(K)$, we may create new components that are unpunctured. We must attach each of those to an adjacent thickened tree $B_{j}^{\prime}$.

We let $C_{0}^{\prime \prime}$ denote the collection of new domains $M$ created by removing the thickened trees $B_{j}^{\prime}$. That is, for each punctured element $L$ of $C_{0}$ that is assigned as $L=L(K)$ for at least one component $K$ of $\bigcup C_{0}^{\prime}$, the components $M$ of $L \backslash \bigcup B_{j}^{\prime}$ are elements of the collection $C_{0}^{\prime \prime}$. We then have the following fact. 
(vii) The elements $M$ of $C_{0}^{\prime \prime}$ that contain no elements of $D(X)$ form a null sequence.

Proof of (vii). Suppose not. Then there are components $M_{1}, M_{2}, \ldots$ that contain no point of $D(X)$ and converge to a nondegenerate continuum $M$. Since the thickened trees $B_{j}^{\prime}$ are formed by adding small one-sided neighborhoods to each side of the trees $B_{j}$, and those neighborhoods contain no points of $D(X)$, we may add those neighborhoods back into the components $M_{i}$ without changing the number of those components, without changing their size in any substantial way, and without changing their limit. We therefore ignore the thickenings and consider the $M_{i}$ 's as components of the complement of the union of the $B_{j}$ 's.

Suppose first that $M$ has a point $p$ that lies in the interior of a small constituent square $T$. Since $\bigcup_{j} B_{j}$ is locally a finite graph away from the edges of the grid, and a finite graph separates an open set locally into only finitely many components, $p \notin \bigcup_{j} B_{j}$. Hence there is a small annulus $A$ surrounding $p$ that contains no point of $\bigcup_{j} B_{j}$. Each $M_{i}$ crosses $A$ in a "large" set, contained in a component of $A \cap U_{0}$ that contains no points of $D(X)$ and no points of $\bigcup_{j} B_{j}$. There are only finitely many such, a contradiction.

Suppose finally that $M$ lies in the 1-skeleton of the grid. Then we may suppose that $M$ contains a nondegenerate interval $I$ of an edge of a small constituent square $T$, and we may assume that each $M_{i}$ also lies in that square. We may take a small rectangular disk neighborhood $A$ of $I^{\prime} \subset I$ in $T$ so that all but finitely many $M_{i}$ cross $A$ from one side to the other near $I^{\prime}$. No point of the larger interval int $(I)$ can lie in $\bigcup_{j} B_{j}$, for most of the $M_{i}$ 's would then have to cross some $B_{j}$, a contradiction. Hence, only large $B_{j}$ 's can come near the smaller interval $I^{\prime}$. Hence $I^{\prime}$ has a neighborhood in $A$ missing $\bigcup_{j} B_{j}$. But, by hypothesis (2), all but finitely many of the components crossing $A$ must contain points of $D(X)$, a contradiction.

This completes the proof of (vii).

Each of the components $M$ just discussed shares an arc with some thickened tree $B_{j}^{\prime}$. We attach each component $M$ to such an adjacent $B_{j}^{\prime}$ along an attaching arc.

STEP 5: Completion of the first null sequence of Peano domains. We have at this point created three null sequences of sets, namely, the components $K$ of $\cup \mathcal{C}_{0}^{\prime}$, the components $B^{\prime}$ of thickened trees, and the unpunctured components $M$ that were formed when the thickened trees were carved out of punctured components of $\mathcal{C}_{0}$. Using the attaching arcs described earlier, we can therefore form a null sequence of domains of the form $V=\operatorname{int}\left(B^{\prime} \cup K_{1} \cup K_{2} \cup \cdots \cup M_{1} \cup M_{2} \cup \cdots\right)$, where $B^{\prime}$ is a thickened tree and the $K$ 's and the $M$ 's are attached to $B^{\prime}$ along attaching arcs. (Each $K_{i}$ and each $M_{i}$ is attached to a unique $B_{j}^{\prime}$. So the $V$ 's are disjoint.) 
(viii) The sets $V$, which obviously form a null sequence of sets, are all Peano domains.

Proof of (viii). We have already noted that $\operatorname{int}\left(B^{\prime}\right)$ is a Peano domain. Each $\operatorname{int}\left(K_{i}\right)$ is a Peano domain because, by hypothesis (2) of this theorem and a two-case argument similar to (iii), it satisfies hypothesis (2) of Theorem 3.2. We see that the sets $M_{j}$ are Peano domains because of the following argument. Suppose there is a disk $D$ such that the components of $M_{j} \cap D$ do not form a null sequence. We let $V_{1}, V_{2}, \ldots$ denote a sequence of components converging to a nondegenerate continuum $V$. We get a contradiction exactly as in the argument for (vii) above.

We now choose, for the closures of $B^{\prime}$ and for the closures of each of the $K_{i}$ 's and each of the $M_{j}$ 's, a continuous surjection from $\mathbb{B}^{2}$ as in condition (3) of Theorem 3.2. By the proof of Theorem 3.2, as noted in the remark following the statement of Theorem 3.2, we may assume that these maps are 1-1 over the attaching arcs. It is thus an easy matter to piece these functions together to get a single continuous surjection from $\mathbb{B}^{2}$ onto the closure of $V=\operatorname{int}\left(B^{\prime} \cup K_{1} \cup K_{2} \cup \cdots \cup M_{1} \cup M_{2} \cup \cdots\right)$ of the kind required by Theorem $3.2(3)$.

This completes the proof of (viii).

STEP 6: Preparing for the next stage of the induction. If $L$ is an element of $\mathcal{C}_{0}$ from which certain thickened trees $B_{i}^{\prime}$ have been removed, then the remaining punctured components all have diameter less than or equal to the mesh of the covering grid. However, they need not form a null sequence. We simply take the union of the interiors of such elements in $\mathbb{R}^{2}$ to form a new open set $U_{1}$. This open set forms the input to the next stage of the induction. We need to verify the following fact:

(ix) The open set $U_{1}$ satisfies the two conditions (1) and (2) with which we began Section 4.

Proof of $(i x)$. The remaining components are all subsets of components of elements of $\mathcal{C}_{0}$, hence have diameter less than or equal to the mesh of the covering grid.

Suppose that $D$ is a disk and $D \cap U_{1}$ has infinitely many large components $M_{i}$ that contain no point of $D(X)$. We may assume $M_{i} \rightarrow M, M$ nondegenerate. We argue again exactly as in the proof of (vii) to obtain a contradiction.

Thus hypothesis (2) is satisfied. Since each component of $U_{1}$ is, by hypothesis, punctured, hypothesis (1) is also satisfied.

This completes the proof of (ix).

STEP 7: The inductive step and the completion of the proof. We now recycle the new open set $U_{1}$ as the set $U_{0}$ of the argument just given, but use 
a grid with much smaller mesh. We repeat this process inductively, infinitely often. The completion of the argument is then clear provided we make the following two remarks:

(x) We may require that the point $p_{i} \in D(X)$ lie in one of the trees constructed before or during the $i$ th stage of the induction.

Proof of $(x)$. We may choose the mesh so small that, if $p_{i}$ has not been used before stage $i$, then $p_{i}$ is the only point of $D(X)$ in a square of the grid and its neighboring squares, all lying in $U_{i}$. We can choose to attach the neighboring squares to the square containing $p_{i}$.

(xi) Eventually, every point $p$ of $\mathbb{S}^{2} \backslash(D(X) \cup B(X))$ lies in the closure of the constructed Peano domains.

Proof of (xi). When squares are sufficiently small, every square containing $p$ misses $D(X) \cup B(X)$. If $p$ has not already appeared in the closure of one of our Peano domains, then $p$ will lie in a component $K$ that contains no point of $D(X)$, hence will be attached to some thickened tree at that stage.

Thus our proof is complete that we can tile the complement of $B(X)$ with a null sequence of disjoint Peano domains. Hence, infinitely many applications of Theorem 3.3 show that $X$ can be deformed by a strong deformation retraction onto a 1-dimensional set.

6. Proof of Theorem 1.4. We are given a planar Peano continuum $M$. We must show that the fundamental group of $M$ embeds in the fundamental group of a 1-dimensional planar Peano continuum $M^{\prime}$.

The construction of the 1-dimensional planar Peano continuum $M^{\prime}$. We shall associate with $M$ a quotient map $\pi: M \rightarrow M^{\prime}$ onto a 1-dimensional Peano continuum $M^{\prime}$ in such a way that each nondegenerate point preimage $\pi^{-1}(x)$, for $x \in M^{\prime}$, is an arc in $M$ with endpoints in $\partial M$.

The vertical decomposition of $M$, and the quotient continuum $M^{\prime}$. Let $V$ be a vertical line that intersects $M$. Let $\mathcal{G}(V)$ denote the set of components of $V \cap M$. Let $\mathcal{G}=\bigcup_{V} \mathcal{G}(V)$. Let $\mathcal{G}_{0}$ be the trivial extension of $\mathcal{G}$ to all of $\mathbb{R}^{2}$. (That is, $\mathcal{G}_{0} \backslash \mathcal{G}$ consists of the singleton sets of $\mathbb{R}^{2} \backslash M$.) Let $\pi: M \rightarrow M^{\prime}=$ $M / \mathcal{G}$ and $\pi^{\prime}: \mathbb{R}^{2} \rightarrow \mathbb{R}^{2} / \mathcal{G}_{0}$ be the associated quotient maps.

Claim 1. The decomposition $\mathcal{G}_{0}$ is cellular and upper semicontinuous, so that $\mathbb{R}^{2} / \mathcal{G}_{0}$ is homeomorphic with $\mathbb{R}^{2}$ by the Moore Decomposition Theorem 2.6. Since each element of $\mathcal{G}$ intersects $\partial(M), M^{\prime}=\pi(M)=\pi^{\prime}(M)$ is nowhere dense in $\mathbb{R}^{2} \sim \mathbb{R}^{2} / \mathcal{G}_{0}$. Consequently, $M^{\prime}$ is a 1-dimensional Peano continuum.

Proof of the claim. Since each element of $\mathcal{G}_{0}$ is a point or an arc, $\mathcal{G}_{0}$ is cellular. Let $g_{1}, g_{2}, \ldots$ be elements of $\mathcal{G}_{0}$ containing convergent sequences 
$x_{i} \rightarrow x$ and $y_{i} \rightarrow y$, with $x_{i}, y_{i} \in g_{i} \in \mathcal{G}_{0}$. If $x \neq y$, then $g_{i}$ must be a vertical interval in $M$ for all $i$ sufficiently large. Thus $x$ and $y$ must be elements of $M$ in the same vertical interval. The vertical intervals $g_{i}$ join $x_{i}$ to $y_{i}$. Hence their limits contain a vertical interval from $x$ to $y$, which must lie in $M$. Thus $x$ and $y$ are in the same element of $\mathcal{G}_{0}$, and $\mathcal{G}_{0}$ is upper semicontinuous.

The remaining assertions of the claim are easily verified.

Claim 2. The projection map $\pi: M \rightarrow M^{\prime}$ induces a map on fundamental groups that is injective. [The proof of this claim will establish Theorem 1.4.]

Proof of the claim. Let $f: \mathbb{S}^{1} \rightarrow M$ be a continuous function such that $f^{\prime}=\pi \circ f: \mathbb{S}^{1} \rightarrow M^{\prime}$ is nullhomotopic in $M^{\prime}$ (that is, there is a map $F^{\prime}: \mathbb{B}^{2} \rightarrow M^{\prime}$ that extends $\left.f^{\prime}\right)$. We must show that $f$ is nullhomotopic in $M$.

Analysis of $f^{\prime}=\pi \circ f: \mathbb{S}^{1} \rightarrow M^{\prime}$. (The analysis is essentially taken from $[3,3.2 .1]$.)

Mapping Analysis Lemma (see [8] and [3, 3.2.1]). Suppose that $f^{\prime}$ : $\mathbb{S}^{1} \rightarrow M^{\prime}$ is a nullhomotopic mapping from the circle $\mathbb{S}^{1}$ into a 1 -dimensional continuum $M^{\prime}$. Then there is an upper semicontinuous decomposition $H$ of $\mathbb{S}^{1}$ into compacta that has the following three properties:

(1) The mapping $f^{\prime}$ is constant on each element of $H$.

(2) The decomposition $H$ is noncrossing. That is, if $h_{1}$ and $h_{2}$ are distinct elements of $H$, then the convex hulls $\mathbf{H u l l}\left(h_{1}\right)$ and $\mathbf{H u l l}\left(h_{2}\right)$ of $h_{1}$ and $h_{2}$ in the disk $\mathbb{B}^{2}$ are disjoint. [Equivalently, $h_{1}$ does not separate $h_{2}$ on $\mathbb{S}^{1}$.]

(3) The decomposition $H$ is filling. That is, the disk $\mathbb{B}^{2}$ is the union of the convex hulls $\mathbf{H u l l}(h)$ of the elements $h \in H$.

Proof. Let $F^{\prime}: \mathbb{B}^{2} \rightarrow M^{\prime}$ be a map that extends $f^{\prime}: \mathbb{S}^{1} \rightarrow M^{\prime}$. We define $H=\left\{h=C \cap \mathbb{S}^{1} \mid \exists x \in M^{\prime}\right.$ such that $C$ is a component of $\left.\left(F^{\prime}\right)^{-1}(x)\right\}$.

It is obvious that $H$ is an upper semicontinuous decomposition of $\mathbb{S}^{1}$ into compacta and that $H$ satisfies conclusion (1) of the Mapping Analysis Lemma. (One can easily check the upper semicontinuity by verifying the following two exercises:

EXERCISE 1. If $f: M_{1} \rightarrow M_{2}$ is a continuous function between compacta, then the components of the point preimages form an upper semicontinuous decomposition $G$ of $M_{1}$. [Indeed, let $x_{i}, y_{i} \in K_{i} \in G$ with $x_{i} \rightarrow x \in K(x) \in G$ and $y_{i} \rightarrow y \in K(y) \in G$. In order to prove the Hausdorff condition, it suffices to show that $K(x)=K(y)$. By Theorems 2.4 and 2.5, we may assume that the sequence $K_{i}$ converges to a continuum $K$. By the continuity of $f, f \mid K$ must be a constant function since each of the 
functions $f \mid K_{i}$ is constant. But $x, y \in K$. Hence $K \subset K(x) \cap K(y)$ and $K(x)=K(y)$.]

EXERCISE 2. If $G$ is an upper semicontinuous decomposition of a compactum $M$ into compact sets, and if $X$ is a compact subset of $M$, then the elements $K \cap X$, for $K \in G$, form an upper semicontinuous decomposition of $X$. [Indeed, proceed in a manner similar to the solution of Exercise 1.])

The proof of conclusion (2) of the Mapping Analysis Lemma is easy. If $h_{1}$ separates $h_{2}$ on $\mathbb{S}^{1}$, and if $h_{1}=C_{1} \cap \mathbb{S}^{1}$ and $h_{2}=C_{2} \cap \mathbb{S}^{1}$, then $C_{1}$ and $C_{2}$ must intersect, a contradiction.

The proof of conclusion (3) requires Lemma 6.1 below, which shows that $\mathbb{S}^{1} / H$ is a contractible set. Knowing that $\mathbb{S}^{1} / H$ is contractible, we argue as follows. Let $H^{\prime}$ be the collection of sets in $\mathbb{R}^{2}$ that are either convex hulls Hull $(h)$ of elements of $h \in H$ or are singleton sets that miss all such convex hulls. Since $H$ is noncrossing, by (2), it follows easily that $H^{\prime}$ is a cellular, upper semicontinuous decomposition of $\mathbb{R}^{2}$. Let $\pi: \mathbb{R}^{2} \rightarrow \mathbb{R}^{2} / H^{\prime} \approx \mathbb{R}^{2}$ denote the projection map. If $H$ were not filling, then the contractible set $\pi\left(\mathbb{S}^{1}\right) \approx \mathbb{S}^{1} / H$ would separate the nonempty sets $\pi\left(\mathbb{R}^{2} \backslash \mathbb{B}^{2}\right)$ and $\pi\left(\mathbb{B}^{2}\right) \backslash \pi\left(\mathbb{S}^{1}\right)$ in $\mathbb{R}^{2} / H^{\prime} \approx \mathbb{R}^{2}$, a contradiction.

Here is the lemma that shows that $\mathbb{S}^{1} / H$ is contractible.

Lemma 6.1. Let $F: \mathbb{B}^{2} \rightarrow M^{\prime}$ be a continuous function from the disk $\mathbb{B}^{2}$ into a 1-dimensional continuum $M^{\prime}$. Define

$$
H=\left\{h=C \cap \mathbb{S}^{1} \mid \exists x \in M^{\prime} \text { such that } C \text { is a component of } F^{-1}(x)\right\} .
$$

Then $H$ is an upper semicontinuous decomposition of $\mathbb{S}^{1}$ into compacta and $\mathbb{S}^{1} / H$ is a 1-dimensional, contractible, planar Peano continuum (that is, $\mathbb{S}^{1} / H$ is a dendrite).

Proof. Each point $p \in \mathbb{B}^{2}$ lies in some component $C$ of some point preimage $F^{-1}(x), x \in M^{\prime}$. We may partially order these components by declaring $C \leq C^{\prime}$ if $C$ lies in the union of $C^{\prime}$ and its bounded complementary domains. By a compactness argument, for each $C$, there is a maximal $C^{\prime}$ with $C \leq C^{\prime}$. We may redefine $F^{\prime}$ so that $F^{\prime}(p)=F^{\prime}(C)$. This modification does not alter the decomposition $H$ of $\mathbb{S}^{1}$. After this modification, the nondegenerate components of point preimages form the nondegenerate elements of a cellular upper semicontinuous decomposition $G$ of $\mathbb{R}^{2}$ (see the two exercises above); and, by the Moore Decomposition Theorem 2.6, the quotient $\mathbb{R}^{2} / G$ is homeomorphic with $\mathbb{R}^{2}$. We denote the quotient map by $\pi^{\prime}: \mathbb{R}^{2} \rightarrow \mathbb{R}^{2} / G \approx \mathbb{R}^{2}$. The modified $F^{\prime}$ factors through the projection $\pi^{\prime} \mid \mathbb{B}^{2}: \mathbb{B}^{2} \rightarrow \mathbb{B}^{2} /\left(G \mid \mathbb{B}^{2}\right)$ :

$$
F^{\prime}: \mathbb{B}^{2} \stackrel{\pi^{\prime} \mid \mathbb{B}^{2}}{\longrightarrow} \mathbb{B}^{2} /\left(G \mid \mathbb{B}^{2}\right) \stackrel{F^{\prime \prime}}{\longrightarrow} M^{\prime}
$$

The image $\pi^{\prime}\left(\mathbb{B}^{2}\right)$ of the disk $\mathbb{B}^{2}$ is contractible because it is a strong deformation retract of the disk $\pi^{\prime}\left(2 \mathbb{B}^{2}\right) \subset \mathbb{R}^{2} / G$. [The set $\pi^{\prime}\left(2 \mathbb{B}^{2}\right)$ is a disk 
since it is a compact set in the plane $\mathbb{R}^{2} / G$ whose boundary is a simple closed curve.]

The image $\pi^{\prime}\left(\mathbb{B}^{2}\right)$ of the disk $\mathbb{B}^{2}$ is 1-dimensional since (i) it admits the mapping $F^{\prime \prime}: \pi^{\prime}\left(\mathbb{B}^{2}\right) \rightarrow M^{\prime}$ into a 1-dimensional space $M^{\prime}$ and the point preimages of $F^{\prime \prime}$ are totally disconnected, while (ii) a map that reduces dimension by $k$ must have at least one point preimage of dimension $k$ ([11, Theorem VI 7]).

The images $\pi^{\prime}\left(\mathbb{B}^{2}\right)$ and $\pi^{\prime}\left(\mathbb{S}^{1}\right)$ are equal for the following reasons. Since $\pi^{\prime}\left(\mathbb{B}^{2}\right)$ is compact and 1-dimensional, the open set $\pi^{\prime}\left(\mathbb{R}^{2} \backslash \mathbb{B}^{2}\right)$ is dense in the plane $\mathbb{R}^{2} / G$. Hence the image of $\pi^{\prime}\left(\mathbb{R}^{2} \backslash \operatorname{int}\left(\mathbb{B}^{2}\right)\right)$ is the entire plane. Consequently, $\pi^{\prime}\left(\mathbb{S}^{1}\right) \supset \pi^{\prime}\left(\mathbb{B}^{2}\right)$. The opposite inclusion is obvious. We conclude that $\pi^{\prime}\left(\mathbb{S}^{1}\right)$ is contractible.

The proof of Lemma 6.1 completes the proof of the Mapping Analysis Lemma.

Completion of the proof that $f: \mathbb{S}^{1} \rightarrow M$ is nullhomotopic. We recall the cellular, upper semicontinuous decomposition $\mathcal{G}$ of $\mathbb{R}^{2}$ that has as its nondegenerate elements the maximal vertical intervals in $M$ and whose quotient $\operatorname{map} \pi: \mathbb{R}^{2} \rightarrow \mathbb{R}^{2} / \mathcal{G}$ takes $M$ onto $M^{\prime}$. We use the Mapping Analysis Lemma to obtain an upper semicontinuous decomposition $H$ of $\mathbb{S}^{1}$ that models the shrinking of $f^{\prime}=\pi \circ f: \mathbb{S}^{1} \rightarrow M^{\prime}$ in the 1 -dimensional set $M^{\prime}$. Since the decomposition $H$ is noncrossing and filling, we may expand this decomposition $H$ to a decomposition $G$ of $\mathbb{B}^{2}$ by taking as elements the convex hulls in $\mathbb{B}^{2}$ of the elements of $H$. The shrinking of $f$ in $M$ will rely on the interplay between the decompositions $\mathcal{G}$ and $G$. We shall use the decomposition $G$ of $\mathbb{B}^{2}$ as a model on which we shall base the construction of a continuous function $F: \mathbb{B}^{2} \rightarrow M$ that extends $f: \mathbb{S}^{1} \rightarrow M$.

If, for each $g \in G, f \mid g \cap \mathbb{S}^{1}$ were constant (as is true for $f^{\prime}$ ), we could simply define $F(g)=f\left(g \cap \mathbb{S}^{1}\right)$. However, this need not be the case. All that we know is that for each $g \in G$, there exists $h(g) \in \mathcal{G}$ such that $f\left(g \cap \mathbb{S}^{1}\right) \subset$ $h(g)$. We need to show how to define $F \mid g: g \rightarrow h(g) \subset M$ in such a way that the union $F=\bigcup\{F \mid g: g \in G\}$ is a continuous extension of $f$.

If $g$ is a single point, then that point lies in $\mathbb{S}^{1}$, and we may define $F(g)=f(g)$.

If $g$ is an interval with its ends in $\mathbb{S}^{1}$, then we extend the map $f \mid \partial g$ linearly to all of $g$.

If $g$ is a disk, then we use an ideal triangulation of $g$ in the following way:

The set $g$ is the convex hull $\mathbf{H u l l}(h)$ of a closed subset $h$ of the unit circle $\mathbb{S}^{1}$. Since $g$ is a disk, $h$ contains at least three points. An ideal triangle is a triangle in $\mathbb{B}^{2}$ that has its vertices on $\mathbb{S}^{1}$. A collection $\left\{T_{i}\right\}$ of ideal triangles is said to be an ideal triangulation of the convex hull $g$ provided that the collection of triangles is locally finite in $\operatorname{int}\left(\mathbb{B}^{2}\right)$, the triangles have 
disjoint interiors, have vertices in $h$, and have union whose intersection with $\operatorname{int}\left(\mathbb{B}^{2}\right)$ is precisely $g \cap \operatorname{int}\left(\mathbb{B}^{2}\right)$.

Triangulation Lemma. If $g=\operatorname{Hull}(h)$ is a disk, then $g$ has an ideal triangulation.

Proof. Every point $x \in \mathbf{H u l l}(h) \cap \operatorname{int}\left(\mathbb{B}^{2}\right)$ has a neighborhood in Hull $(h)$ that is in the convex hull of a finite collection of points in $h$. [Hint: every point of a convex hull lies in the hull of a finite subset; consider separately the case where the point is in the interior or on the boundary of such a finite polygon.] Hence, every compact subset of $\operatorname{Hull}(h) \cap \operatorname{int}\left(\mathbb{B}^{2}\right)$ is in the convex hull of a finite collection of points in $h$.

Let $C_{1} \subset C_{2} \subset \cdots$ be an exhaustion of $\mathbf{H u l l}(h) \cap \operatorname{int}\left(\mathbb{B}^{2}\right)$ by compact sets, and let $F_{1} \subset F_{2} \subset \cdots$ be finite subsets of $h$ such that $C_{i} \subset \mathbf{H u l l}\left(F_{i}\right)$. It suffices to show that any ideal triangulation $T_{i}$ of $\mathbf{H u l l}\left(F_{i}\right)$ extends to an ideal triangulation $T_{i+1}$ of $\mathbf{H u l l}\left(F_{i+1}\right)$, for then we may take $T(X)=$ $\bigcup_{i=1}^{\infty} T_{i}$.

To extend $T_{i}$ to $T_{i+1}$, it suffices to see that we can add one point $p$ at a time to $F_{i}$. Since each edge of $T_{i}$ separates $\mathbb{B}^{2}$, the domain of $\mathbb{B}^{2} \backslash\left|T_{i}\right|$ that contains $p$ is bounded by a single edge $r s$ of $T_{i}$ followed by an arc of $\mathbb{S}^{1}$ that contains $p$. We simply add the triangle $\operatorname{prs}$ to $T_{i}$.

Note that, since every compact set $C_{i}$ in $g \cap \operatorname{int}\left(\mathbb{B}^{2}\right)$ is actually covered by a finite subcollection of the triangles $T_{j}$, the collection is locally finite in $\operatorname{int}\left(\mathbb{B}^{2}\right)$.

This completes the proof of the Triangulation Lemma.

With the Triangulation Lemma in hand, we are ready to define $F \mid g$ : $g \rightarrow M$, for the case where $g$ is a disk.

In this case, we note that $h=g \cap \mathbb{S}^{1}$ is a compact set having at least three points. Hence, by the Triangulation Lemma, $g$ has an ideal triangulation $T(g)$. We define $F$ on $g \cap \mathbb{S}^{1}$ to equal $f$. On each triangle $t_{i}$ of $T(g)$, we define $F$ to be the linear extension of $f$ restricted to the three vertices of $t_{i}$.

Proof that $F \mid g$ is continuous for each $g \in G$. If $F \mid g$ is not continuous, then there are $x_{i} \rightarrow x$ in $g$ and $\varepsilon>0$ such that $d\left(F\left(x_{i}\right), F(x)\right) \geq \varepsilon$ for all $i$. Since $F\left|g \cap \mathbb{S}^{1}=f\right| g \cap \mathbb{S}^{1}$ is continuous, we may assume that each $x_{i}$ lies in $\operatorname{int}\left(\mathbb{B}^{2}\right)$. Since $F$ is continuous on any finite union of triangles of $T(g)$ and since $T(g)$ is locally finite in $\operatorname{int}\left(\mathbb{B}^{2}\right)$, we may assume that $x \in h=g \cap \mathbb{S}^{1}$ and that $x_{1}, x_{2}, \ldots$ come from distinct triangles of $T(g)$. Since these triangles cannot accumulate at any interior point of $\mathbb{B}^{2}$, they must, in fact, have diameter going to 0 and approach $x$. But then their vertices approach $x$ and, by linearity, their images approach $F(x)$, a contradiction. Hence $F \mid g$ is continuous. 
Proof that $F$ is continuous. If $F$ is not continuous, then there are $x_{i} \rightarrow x$ in $\mathbb{B}^{2}$ and $\varepsilon>0$ such that $d\left(F\left(x_{i}\right), F(x)\right) \geq \varepsilon$ for all $i$. Since, for each $g \in G$, $F \mid g$ is continuous, we may assume that $x_{1}, x_{2}, \ldots, x$ all come from distinct elements $g_{1}, g_{2}, \ldots, g$ of $G$. By continuity of $F \mid \mathbb{S}^{1}=f$, we may ignore those $x_{i}$ in $\mathbb{S}^{1}$. Hence, we may assume that $x_{i} \in \operatorname{int}\left(\mathbb{B}^{2}\right)$, and that $g_{i}$ is either an arc $t_{i}$ or a disk, one of whose triangles $t_{i}$ contains $x_{i}$. If the $t_{i}$ approach $x$, then $x \in \mathbb{S}^{1}$, the vertices of the $t_{i}$ approach $x$, and the images of the $t_{i}$ approach $F(x)$ by linearity and the continuity of $F \mid \mathbb{S}^{1}=f$. Otherwise, we may assume that the $t_{i}$ approach an edge $t$ of $g$ that contains $x$. Again, their vertices approach the vertices of $t$, and the continuity of $F \mid \mathbb{S}^{1}=f$ and linearity imply that $F\left(x_{i}\right) \rightarrow F(x)$, a contradiction. We conclude that $F$ is continuous.

This completes the proof of Theorem 1.4.

We recall the corollary and question associated with Theorem 1.4:

Corollary 1.5. If $M$ is a planar Peano continuum, then the fundamental group of $M$ embeds in an inverse limit of finitely generated free groups.

Proof. This theorem is well-known for 1-dimensional continua. See, for example, [8] and [4].

QUESTION 1.6. If $M$ is a planar Peano continuum whose fundamental group is isomorphic with the fundamental group of some 1-dimensional planar Peano continuum, is it true that $M$ is homotopically 1-dimensional?

It is not difficult to see that the projection that we have given that takes $M$ onto $M^{\prime}$ does not give a surjection on fundamental groups if $M$ is not homotopically 1-dimensional. The key issue to resolve here is whether an arbitrary group embedding into the group of a 1-dimensional continuum can always be induced by a continuous map.

Corollary 1.7. If $M$ is a planar Peano continuum, $f: \mathbb{S}^{1} \rightarrow M$ is a loop in $M$, and $f$ is nullhomotopic in every neighborhood of $M$ in $\mathbb{R}^{2}$, then $f$ is nullhomotopic in $M$.

Proof. It follows easily that $f^{\prime}: \mathbb{S}^{1} \rightarrow M^{\prime}$ is nullhomotopic in each neighborhood of $M^{\prime}$ in $\mathbb{R}^{2}$. But it is well-known [8], [3] that this implies that $f^{\prime}$ is nullhomotopic in $M^{\prime}$. Thus the argument of Theorem 1.4 applies to show that $f$ is nullhomotopic in $M$.

For results that strengthen the last corollary, see [9].

\section{References}

[1] P. S. Aleksandrov, Combinatorial Topology, Vol. 1, Graylock Press, Rochester, NY, 1956.

[2] J. W. Cannon, The recognition problem: what is a topological manifold?, Bull. Amer. Math. Soc. 84 (1978), 832-866. 
[3] J. W. Cannon and G. R. Conner, On the fundamental groups of one-dimensional spaces, Topology Appl. 153 (2006), 2648-2672.

[4] J. W. Cannon, G. R. Conner and A. Zastrow, One-dimensional sets and planar sets are aspherical, ibid. 120 (2002), 23-45.

[5] R. Charlip, Fortunately, Aladdin Paperbacks of Simon and Schuster, New York, 1964.

[6] J. B. Conway, Functions of One Complex Variable II, Springer, New York, 1995.

[7] R. J. Daverman, Decompositions of Manifolds, Pure Appl. Math. Ser. 124, Academic Press, New York, 1986.

[8] M. L. Curtis and M. K. Fort Jr., Homotopy groups of one-dimensional spaces, Proc. Amer. Math. Soc. 8 (1957), 577-579.

[9] H. Fischer and A. Zastrow, The fundamental groups of subsets of closed surfaces inject into their first shape groups, Algebr. Geom. Topol. 5 (2005), 1655-1676.

[10] J. G. Hocking and G. S. Young, Topology, Dover Publ., 1988.

[11] W. Hurewicz and H. Wallman, Dimension Theory, Princeton Univ. Press, Princeton, 1941.

[12] U. Karimov, D. Repovš, W. Rosicki and A. Zastrow, On two-dimensional planar compacta which are not homotopically equivalent to any one-dimensional compactum, Topology Appl. 153 (2005), 284-293.

[13] R. L. Moore, Concerning a set of postulates for plane analysis situs, Trans. Amer. Math. Soc. 20 (1919), 169-178.

[14] - Concerning upper semi-continuous collections of continua, ibid. 27 (1925), 416428.

[15] -, Foundations of Point Set Theory, rev. ed., Colloq. Publ. 13, Amer. Math. Soc., Providence, RI, 1962.

[16] J. R. Munkres, Topology, 2nd ed., Prentice-Hall, Upper Saddle River, NJ, 2000.

[17] E. H. Spanier, Algebraic Topology, Springer, New York, 1989.

[18] G. T. Whyburn, Topological characterization of the Sierpinski curve, Fund. Math. 45 (1958), 320-324.

[19] —, Analytic Topology, Colloq. Publ. 28, Amer. Math. Soc., Providence, RI, 1963.

[20] - Topological Analysis, rev. ed., Princeton Math. Ser. 23, Princeton Univ. Press, Princeton, NJ, 1964.

[21] R. L. Wilder, Topology of Manifolds, Colloq. Publ. 32, Amer. Math. Soc. Providence, RI, 1963.

[22] A. Zastrow, Planar sets are aspherical, Habilitationsschrift, Ruhr-Universität Bochum, 1997-1998.

Department of Mathematics

Brigham Young University

Provo, UT 84602, U.S.A.

E-mail: cannon@math.byu.edu

conner@math.byu.edu

Received 1 July 2004;

in revised form 31 March 2007 